\title{
Modeling and Simulation of Nonlinear Dynamics of Flapping Wing Micro Air Vehicles
}

\author{
Christopher T. Orlowski* and Anouck R. Girard亡 \\ University of Michigan, Ann Arbor, Michigan 48109
}

DOI: $10.2514 / 1 . J 050649$

\begin{abstract}
The derivation of the nonlinear dynamics of flapping wing micro air vehicles is presented. Simulation results investigate differences in the position and orientation of the body due to differing wing masses and aerodynamic modeling choices. The flapping wing micro air vehicle is modeled as a system of three rigid bodies: a body and two wings. The mass of the wings, and their associated mass and inertial effects on the body, are thoroughly analyzed and included. Simulations are compared with previous modeling efforts, which neglected the wings' mass and the associated inertial coupling effects on the body. Simulations show a qualitative consistency for the nonlinear model with wing effects when different aerodynamic models are chosen as inputs. Simulation results show a significant difference in the model behavior when the mass of the wings, initially set at $5.7 \%$ of the body mass, is included versus when the mass is neglected. As the mass of the wings is decreased, the simulation results of the model with wing effects approach the results when the standard aircraft model is used. Simulations lead to the conclusion that the mass effects of the wings are important for dynamics, stability, and control analyses.
\end{abstract}

\section{Nomenclature}

$B \quad \hat{b}_{x} \quad=$ central body-fixed reference frame

$\hat{b}_{x}, \hat{b}_{y}, \hat{b}_{z}=$ axes for the body frame

$\bar{F}_{\text {aero }} \quad=$ aerodynamic forces expressed in the body frame

$F_{N, k}, F_{T, k}=$ aerodynamic normal and tangential forces, expressed in the respective wing frame

$F_{x}, F_{y}, F_{z}=$ components of the resultant aerodynamic forces, expressed in the body frame

$F_{x, k}, F_{y, k}, \quad=$ the resultant aerodynamic forces produced by the

$F_{z, k} \quad$ individual wings, expressed in the body frame

$\overline{\mathbf{H}}_{i} \quad=$ angular momentum of the $i$ th rigid body

$\mathbf{I}_{i} \quad=$ inertia tensor for the $i$ th rigid body

$L, M, N=$ components of the resultant aerodynamic moments, expressed in the body frame

$L_{\mathrm{sp}} \quad=$ stroke plane frame for the left wing

$L_{w} \quad=$ wing fixed frame for the left wing

$L_{x}, L_{y}, L_{z}=$ components of the left wing joint reference vector, $\bar{r}_{L}$

$\bar{M}_{\text {aero }} \quad=$ aerodynamic moments expressed in the body frame

$M_{g, k} \quad=$ moments due to gravity and the position of the wings

$m_{i} \quad=$ mass of the $i$ th rigid body

$p, q, r=$ angular velocity components of central body in body frame

$\overline{\mathbf{p}}_{i} \quad=\quad$ linear momentum of the $i$ th rigid body

$p_{\mathrm{LW}}, q_{\mathrm{LW}}, \quad=$ angular velocity components of left wing in stroke

$r_{\mathrm{LW}} \quad$ plane frame

$p_{\mathrm{RW}}, q_{\mathrm{RW}},=$ angular velocity components of right wing in

$r_{\mathrm{RW}} \quad$ stroke plane frame

$Q_{j} \quad=$ generalized force/moment for the $j$ th generalized coordinate $\bar{\beta}_{i j}$

Received 17 May 2010; revision received 8 September 2010; accepted for publication 10 December 2010. This material is declared a work of the U.S. Government and is not subject to copyright protection in the United States. Copies of this paper may be made for personal or internal use, on condition that the copier pay the $\$ 10.00$ per-copy fee to the Copyright Clearance Center, Inc., 222 Rosewood Drive, Danvers, MA 01923; include the code 0001-1452/ 11 and $\$ 10.00$ in correspondence with the CCC.

${ }^{*} \mathrm{Ph} . \mathrm{D}$. Candidate, Aerospace, Robotics, and Controls Laboratory, Department of Aerospace Engineering. Senior Member AIAA.

Assistant Professor, Aerospace, Robotics, and Controls Laboratory, Department of Aerospace Engineering. Associate Member AIAA.
$\mathbf{R}_{B}$

$\mathbf{R}_{R}, \mathbf{R}_{L}$

$R_{\mathrm{sp}}$

$R_{w}$

$R_{x}, R_{y}, R_{z}$

$\underset{\mathbf{R}_{\zeta_{k}}}{\mathbf{R}_{\alpha_{k}}, \mathbf{R}_{\delta_{k}},}$

$\mathbf{R}_{\beta_{k}}$

$\bar{r}_{R}, \bar{r}_{L}$

$u, v, w$

$\overline{\mathbf{v}}_{i}$

$\hat{x}_{\mathrm{sp}, k}, \hat{y}_{\mathrm{sp}, k}$,

$\hat{z}_{\mathrm{sp}, k}$

$\hat{x}_{w, k}, \hat{y}_{w, k}$,

$\hat{z}_{w, k}$

$\alpha_{R}, \alpha_{L}$

$\dot{\alpha}_{R}, \dot{\alpha}_{L}$

$\beta_{R}, \beta_{L}$

$\bar{\gamma}_{i j}$

$\delta_{R}, \delta_{L}$

$\dot{\delta}_{R}, \dot{\delta}_{L}$

$\zeta_{R}, \zeta_{L}$

$\dot{\zeta}_{R}, \dot{\zeta}_{L}$

$\theta$

$=$ rotation matrix from the inertial frame to the body frame

$=$ rotation matrix from the right (left) stroke plane frame to the right (left) wing frame

$=$ stroke plane frame for the right wing

$=$ wing fixed frame for the right wing

$=$ components of the right wing joint reference vector, $\bar{r}_{R}$

$=$ rotation matrices for the individual wing angles

$=$ rotation matrix from the body frame to the stroke plane frame

$=$ position vector of the wing joints with respect to the origin of the body frame

$=$ translational velocity components of central body in body frame

$=$ velocity vector for the $i$ th rigid body

$=$ axes for the stroke plane reference frames

$=$ axes for the wing reference frames

$=$ angle of attack of the right and left wings

$=$ time derivative of the angle of attack

$=$ stroke plane angle, relative to the $\hat{b}_{y}$ axis of the body frame

$=$ angular velocity coefficient of the $i$ th body with respect to the $j$ th quasi velocity

$=$ velocity coefficient of the $i$ th body with respect to the $j$ th quasi velocity

$=$ deviation (elevation) angle of the right and left wings

$=$ time derivative of the deviation angle

= flap (sweep) angle of the right and left wings

$=$ time derivative of the flap angle

$=$ pitch angle of the central body

$=$ position vector from the wing joint to the aerodynamic center of pressure

$\bar{\rho}_{c i} \quad=$ position vector from the wing joint to the respective wing's center of mass in body frame

$\bar{\rho}_{c i \text { sp }}=$ position vector from the wing joint to the respective wing's center of mass in stroke plane frame

$\bar{\rho}_{c i, w} \quad=$ position vector from the wing joint to the respective wing's center of mass in the wing frame 

$\rho_{i}$
$=$ distance of center of mass of the wing from the wing root in the wing frame
$\phi \quad=$ roll angle of the central body
$\psi \quad=$ yaw angle of the central body
$\bar{\omega}_{i} \quad=$ angular velocity vector for the $i$ th rigid body with respect to the $B$ frame
$\bar{\omega}_{i, \mathrm{sp}} \quad=$ angular velocity vector for the right and left wings with respect to the stroke plane frame

Subscripts

$\begin{array}{lll}i & = & \text { rigid body number } \\ j & = & \text { coordinate number } \\ k & & \text { right wing, } R, \text { or left wing, } L\end{array}$

\section{Introduction}

$\mathbf{T}$ HE potential benefits for insectlike flapping wing micro air vehicles are numerous. The hovering ability of insects, coupled with the ability for a quick transition to forward flight, provide an ideal reconnaissance platform for search and rescue, law enforcement, and military efforts. Studies of the dynamics of flapping wing micro air vehicles is generally focused on using the standard aircraft six degrees of freedom equations found in many flight dynamics textbooks, e.g., the treatment by Etkin and Reid in [1]. The wings of insects are generally less than $5 \%$ of their total body mass. For example, the mass of the wings of the desert locust Schistocerca gregaria is less than $4 \%$ of the total body mass [2]. In the study of hawkmoths by Willmott and Ellington [3], the wing mass of the specimens studied ranged between 4.8 and $5.8 \%$. In many dynamics studies, the mass of the wings, and the associated inertial effects, are neglected due to the wings' small mass relative to the rest of the body. The kinematics of insect wings are widely studied, as well as the aerodynamic mechanisms generating the aerodynamic forces and moments necessary for various flight conditions. Ellington produced a seminal study of hovering insect flight in [4]. The morphological relationship between the wings and the body of insects is studied and published for various species, such as hawkmoths in [3], dragonflies in [] ], and bumblebees in [6]. The studies of insect flight provide a good basis for designing flapping mechanisms and wing strokes to effectively produce biomimetic robots [7].

Sun et al. showed in [8] that although the effects of the wings on the body of the insect are small, there still exists a per flapping cycle change in the orientation of the body due to the inertial coupling of the wings. We will present the derivation of the nonlinear dynamics of a flapping wing micro air vehicle (FWMAV). The FWMAV is modeled as a system of three rigid bodies. The wings are each allowed three degrees of freedom relative to the stroke plane. The stroke plane is defined as the plane that defines the mean motion of the wing. The wings have freedom to deviate from the stroke plane, pitch about the wing root, and flap through the stroke plane. The development of the flight dynamics model will provide a basis for further studies of the dynamics, stability and control of FWMAVs. The model will enable the study of many of the open questions regarding FWMAVs: dynamic sensitivity to aerodynamic modeling, general stability (e.g., not tied to a specific insect species or aerodynamic model), ability reject to disturbances (e.g., wind gusts), and controllability studies with the wing effects included, to name a few.

The paper will be organized in the following manner. Section II will present a literature review focusing on previous efforts into deriving and presenting the equations of motion for FWMAVs. Section III will present the derivation of the nonlinear flight dynamics. Section IV will present the dynamics model that is the basis for much of the analysis of the dynamics and stability of FWMAVs. Section $\mathrm{V}$ will present the chosen parameters for the simulations. Section $\underline{\underline{V} I}$ will present simulation results for a normal hovering mode and a water-treading hovering mode. Simulations of the full nonlinear model, to be developed in this paper, will be compared with simulations of a six degrees of freedom aircraft model that neglects the mass of the wings and a six degrees of freedom aircraft model that only includes the gravity moment of the wings on the central body. Simulations will show that the quantitative performance of the model is dependent on the choice of aerodynamic model, but the dynamic model exhibits a qualitative consistency for different aerodynamic models. Additional simulations will show that as the mass of the wings is decreased, relative to the mass of the central body, the behavior of nonlinear model with wing effects approaches the behavior of the standard aircraft model.

\section{Literature Review}

The literature review focuses on previous work on the dynamics of FWMAVs. For further reference, the following set of equations, defined in a body frame fixed to the central body, will be referred to as the standard aircraft equations of motion:

$$
\begin{aligned}
& \dot{\overline{\mathbf{v}}}_{b}=\frac{1}{m_{b}}\left(\bar{W}_{b}+\bar{F}_{\text {aero }}\right)+\bar{\omega}_{b} \times \overline{\mathbf{v}}_{b} \\
& \dot{\bar{\omega}}_{b}=\mathbf{I}_{b}^{-1}\left(\bar{\omega}_{b} \times \mathbf{I}_{b} \bar{\omega}_{b}+\bar{M}_{\text {aero }}\right)
\end{aligned}
$$

In Eq. (1), $\bar{\omega}_{b}$ denotes the angular velocity of the central body, $\overline{\mathbf{v}}_{b}$ is the translational velocity of the central body, $m_{b}$ is the mass of the central body, $\mathbf{I}_{b}$ is the inertia tensor of the central body about its respective center of mass, and $\bar{W}_{b}$ is the weight vector of the body expressed in a body frame. The standard aircraft model neglects the inertial and mass effects of the wings on the central body. The wings' motion is solely included to generate aerodynamic forces and moments on the central body. The set of equations in Eq. (1) are simulated when combined with an aerodynamic model using the traditional equations of motion for six degrees of freedom, rigid body flight.

Preliminary work into the flight dynamics of FWMAVs is taken from the analysis of the flight mechanics and stability of specific insect species. In [2], Taylor and Thomas use the linearized equations of rigid body motion, available in [1] , to study the flight stability of the desert locust Schistocerca gregaria. The mass of the wings, and the associated gyroscopic terms, are neglected due to the assumption that the wings beat fast enough to not excite the rigid body modes of the central body. The stability derivatives are obtained from experimental methods. The authors acknowledge that the linearized system may not be the best approximation of the flight behavior of the desert locust. Taylor and Thomas state that the rigid body approximation is only valid if the wing-beat frequency is at least 10 times higher than the fastest rigid body mode. Taylor et al. expand the work presented in [2] to nonlinear longitudinal dynamics of the desert locust in [9]. The authors present a combination of stability and control derivatives obtained from experiments. The derivatives are combined due to the inability to distinguish between active and passive stability in insects. The derivatives are then used for a nonlinear time-periodic (NLTP) model of the longitudinal dynamics. Both the linear and NLTP model neglect the mass effects of the wings and are longitudinal models only.

In [10], Sun and Xiong use the same rigid body approximation as in [2] to analyze the hovering flight stability of a bumblebee. The stability derivatives are obtained from computational fluid dynamics using flight data from [6]. The analysis results in the determination that, based on the linearized system, the bumblebee has an unstable oscillatory mode for the longitudinal axis. In an open loop setting, the aerodynamic pitching moment destabilizes the longitudinal axis. Improper phasing between the pitching and flapping motion of the wings will enhance the destabilizing effects of the pitching moment.

The standard aircraft model is used to analyze the stability of the flight dynamics of an ornithopter by Dietl and Garcia in [11]. The vehicle dynamics model is presented along with the aerodynamic model, which is developed in [12]. Dietl and Garcia present the longitudinal dynamics, trim solutions and a limit cycle. The longitudinal dynamics are decoupled from the lateral dynamics. In addition to an analysis of the dynamics of the vehicle, control solutions are presented, based on the discrete-time eigenvalues resulting from the periodic solution. The analysis of the dynamics neglects the inertial effects of the wings on the central body and limits 
the wings to birdlike flapping: one degree of freedom with passive rotation of the wings due to aerodynamic pressure on the wing.

In $[13,14]$, Doman et al. present modeling and control of a FWMAV based on the "RoboFly" developed by Wood and presented in [15]. The aerodynamic model used in the simulations is developed in [13] and based on the work of Sane and Dickinson in [16]. The cycle-averaged aerodynamic forces and moments are presented in detail, along with calculation of the control derivatives based on the dynamic and aerodynamic models. The authors present a method of controlling the six degrees of freedom of the central body through the use of split-cycle control. Doman et al. neglect the mass of the wings and their associated effects. Khan and Agrawal, in [17], develop the equations of motion for a FWMAV based on the standard aircraft model. The mass of the wings are neglected, but the wings are given three degrees of freedom relative to the central body and related to the stroke plane by 2-3-1 Euler angles. An aerodynamic model is developed, based on [16], that includes rotational and leading edge vortex effects. The coefficients for the aerodynamic model are determined from a robotic flapper. The wing dimensions from the robotic flapper and the mathematical model are used to present simulations of the FWMAV in a hover condition.

Deng et al. using the standard aircraft model and time-averaged forces and moments to derive a switching controller for a biomimetic insect in [18]. The controller is used, with success, to control the biomimetic insect in the vicinity of a hover condition. Schenato et al. present a controllability study of a biomimetic insect using the standard aircraft equations in [19]. In [20,21], Deng et al. develop a mathematical model for the dynamics of a FWMAV. The authors develop the dynamics, based on the standard model presented in Eq. (1). An aerodynamic model is developed and based on the "Robot Fly" experiments by Sane and Dickinson in [16]. The dynamics of the actuators are included, as well as the required sensors for effective flight. The wings are given three degrees of freedom, but parameterized into one single degree of freedom. The averaged dynamics and aerodynamic inputs are presented and used as a basis for controller and sensor design. Using averaging and linear quadratic regulator theory, a control strategy for ensuring proper stroke kinematics is presented resulting in good tracking of a reference flight condition.

From an extensive review, we discovered very few models of the flight dynamics of FWMAVs that treated the inertial/mass effects of the wings on the central body, and by extension, the entire system. Lasek and Sibilski [22] and Buler et al. [23] derive the equations of motion for a FWMAV using the Gibbs-Appel Equations. Lasek and Sibilski develop a simulation architecture in [22] and limit the wings to two degrees of freedom: flapping and lagging. Alternatively, flapping is the deviation angle and lagging is the flapping (sweep) angle. The feathering, or pitch angle, remains fixed in the study. Buler et al. [23] model a FWMAV with two degrees of freedom for each wing: sweep in the stroke plane and an angle of attack relative to the stroke plane. The authors derived a flight dynamics model with six degrees of freedom for the central body and two holonomically constrained degrees of freedom for each wing, resulting in a system with 10 degrees of freedom. Buler et al. use the model to numerically calculate a Jacobian linearization of the system around a desired trajectory and present a linear quadratic regulator control solution to track the trajectory. In [24], Jackson et al. present a trajectory optimization problem for a FWMAV. The FWMAV is modeled as a system of three bodies, but the central body is modeled as a point mass. The inertial effects of the wings are included, but without the presence of a rigid body for the central body, the inertial coupling between the wings and the central body is not accounted for in the simulations and trajectory optimization problem.

In [25], Grauer and Hubbard derived the equations of motion of an ornithopter using the Boltzman-Hamel equations and five rigid bodies: one for the central body, one for each wing, and two determining the linkage for the tail. The work is motivated to provide control of a relatively larger vehicle; namely an ornithopter with a $4 \mathrm{ft}$ wingspan. Each linked rigid body is allowed one degree of freedom. The angle of attack of the wings is due to passive rotation and a quasisteady aerodynamic model is used for simulation efforts. Addi- tionally, the equations of motion are transformed into a form found often in spacecraft and robotics control in order to allow for the integration of nonlinear control techniques.

Bolender derived the equations of motion for a FWMAV using Kane's Equations in [26]. The novel approach conducted by Bolender is to derive the equations of motion with four rigid bodies: a central body, a tail, and two wings. The tail is used for pitch control of the central body. Bolender includes the derivation of the inertial and active forces, necessary for Kane's equations, but limits the derivation of the wings. The wings are allotted two degrees of freedom, but the effects of the wings on the central body can be reconfigured through tilting of the stroke plane. The simulations presented in [26] are for the dynamic model with wing effects included and are simulated in an open loop fashion. Furthermore, the presented simulations include the motion of the tail. The equations show that without control, the magnitude of the pitch velocity of the central body steadily increases.

Sun et al. present a derivation of the equations of motion for an insect in [27]. The wings are modeled with three degrees of freedom. The motion of the wings is prescribed and the wings are not considered to be separate degrees of freedom. The equations of motion are derived using Eulerian techniques. Sun et al. use various simplifying assumptions to arrive at the standard aircraft equations of motion. The remainder of [27] presents a linearization of the equations of motion about a hovering condition. The stability derivatives for the hover condition are obtained by coupling the equations of motion with the Navier-Stokes equations. The eigenvalues and eigenvectors for the linearization are obtained for four different insects. The authors conclude that the rigid body assumption is valid for small insects, but not necessarily valid for larger insects such as hawkmoths and craneflies.

In [28], Wu et al. present simulations of the equations of motion, previously derived by Sun et al. in [27]. The authors present a method of solving the required parameters for a hover condition by coupling the equations of motion with the Navier-Stokes equations. The required parameters for hover are solved for using a "shooting" method. Results are presented for a dronefly and a hawkmoth. The hover condition is solved for the longitudinal equations of motion; the lateral motion of the insect model is neglected. The results for the hover condition are qualitatively consistent with results obtained from biological studies of droneflies and hawkmoths.

In [29], Gebert et al. derive the equations of motion for a FWMAV using Newtonian methods, which requires the calculation of the constraint forces between the wings and the body. The wings are not neglected, but simulations are not presented to validate the efforts. Furthermore, Sun et al. claim in [27] that the equations of motion derived in [29] contain errors and cannot be used. Dickson et al. present simulation efforts for a FWMAV that includes the mass effects of the wings in [30]. The method chosen uses physics engine software, similar to that used to make video games and animated features, to model the wings and body. Simulations are presented using the physics engine. If equations of motion were developed, which may be important for the development of relevant control algorithms, the equations are not presented.

The many models previously discussed in the literature review are summarized in Table 1 . The dynamics of FWMAVs is widely studied from the aspect of a rigid body approximation. However, studying the multiple-body nonlinear system, with gyroscopic effects from the wings, has not been widely reported. In [26], Bolender makes the claim that the effects of the wings need to be included for proper control studies. The aerodynamic models used by Doman et al. in $[\underline{13}, 14]$, Schenato et al. in [19], and Deng et al. in [20,21] are based on the experiments of Sane and Dicksinson in [16]. The lift and drag coefficients used by Doman et al. are derived from model wing experiments in an oil tank. The model wing is based off of the common fruit fly, Drosophila melanogaster, but the wings are scaled to a semispan of $25 \mathrm{~cm}$ and mean chord of $6.7 \mathrm{~cm}$. Sane and Dickinson conducted their experiments at a Reynolds number of 115 , whereas hawkmoths operate in a flow regime with a Reynolds number of approximately 6000-8000 [3, 31]. The dynamics models derived by Taylor and Thomas in [2], Sun and Xiong in [10], and by 
Table 1 Summary table of dynamics models

\begin{tabular}{|c|c|c|c|c|}
\hline Authors & Dynamics model & Wing effects & Aerodynamics & Applicability \\
\hline Taylor and Thomas [2] & Linear & No & Experimental & Desert locusts \\
\hline Sun and Xiong [10] & Linear & No & CFD & Honeybees \\
\hline Doman et al. $[13,14]$ & Nonlinear & No & Quasi steady/blade element & Robot Fly \\
\hline Deng et al. [18] & Nonlinear & No & Quasi steady/blade element & Small wing mass \\
\hline Bolender [26] & Nonlinear & Yes (two degrees of freedom per wing) & Berman and Wang [12] & No limitations \\
\hline Sun et al. [27] & Nonlinear & Derived (not simulated) & Navier-Stokes & No limitations \\
\hline Buler et al. [23] & Nonlinear & Yes (two degrees of freedom per wing) & Not specified & Ornithopters \\
\hline Grauer and Hubbard [25] & Nonlinear & Yes (one degree of freedom per wing) & Quasi steady & Ornithopters \\
\hline Orlowski et al. $[36,37]$ & Nonlinear & Yes (three degrees of freedom per wing) & Berman and Wang [12] & No limitations \\
\hline
\end{tabular}

Doman et al. in $[13,14]$ are directly tied to the aerodynamics models presented in the papers. Especially in $[2,10]$, the dynamic and aerodynamic results are specifically tied to the insect species presented in the specific studies.

In regard to the other studies presented, the work is not tied to a specific aerodynamic model. The aerodynamic models are chosen as inputs. In [19-21], the control results are tied to the aerodynamic model, but the dynamics presentations allows for the implementation of different aerodynamic models. However, the presented results neglect the mass of the wings. The work by Lesak and Sibiliski in [22], Buler et al. in [23], and Grauer and Hubbard in [25] include the mass effects of the wings, but the formulation is limited to ornithopter flight and not true insect flight. Bolender, in [26], included the wing effects in the derivation and simulations, but the wings only have two degrees of freedom relative to the stroke plane. Shyy et al. state that the third degree of freedom, relative to the stroke plane, is important for the transition from hover to forward flight for insectlike flapping [31]. The results presented by Sun et al. in [27] are presented without the mass effects of the wings and the linear solutions are based upon the calculations performed using the Navier-Stokes equations. The aerodynamic model used in [27] is most likely more accurate, but the method is computationally expensive.

Throughout all of the previous references discussed, the work is presented for a single aerodynamic model and the effects of the mass of the wings is either included, or neglected. An analysis of how the wings may affect the central body is largely absent from the literature. Furthermore, since every aerodynamic model is an approximation (of varying degrees of fidelity) of the actual aerodynamic forces and moments generated by the wings, there has not been an attempt to quantify, or qualify, the effects of choosing a different aerodynamic model. The main issue is the availability of computationally efficient aerodynamic models for dynamics, control, and stability studies. The most accurate modeling of flapping wings is obtained from computational fluid dynamics methods, but to obtain data is computationally expensive. Tying these models to a dynamics model may further increase the computational time. Quasi-steady/blade-element models are computationally inexpensive, but their accuracy is debatable. The model used for previous simulations, developed by Berman and Wang in [12], achieves decent accuracy for calculations when compared with the computational fluid dynamics (CFD) efforts by Sun and $\mathrm{Du}$ in [32]. However, the point of this study is to determine the inertial effects of the wings on the dynamics of the central body for future stability and control studies. A quasi-steady aerodynamic model will suffice. Specifically, the goal is to develop and analyze model that includes the inertial coupling effects of the wings on the central body, due to the continuous motion of the wings. The model must be able to replicate true insect flight with three degrees of freedom relative to a stroke plane. All effects of the wings on the body, and vice versa, are included in the derivation. None of the effects will be neglected or simplified. The model will provide a basis for the analysis of the system response due to aerodynamic inputs, stability, and control strategies.

\section{Derivation of the Equations of Motion}

\section{A. Method}

We now present the development of a dynamic model that encompasses the inertial effects of the wings, as well as allowing three degrees of freedom for the wings relative to the stroke plane. The method chosen for the derivation is D'Alembert's Principle for Multiple Rigid Bodies, alternatively presented as the "general form of the equations of motion for multiple rigid bodies" in [33]. The derivation method is presented in [33-35]. The chosen method is a hybrid of Euler and Lagrange techniques and is akin to Kane's equations and the Gibbs-Appel Equations [35]. The flight dynamics model is previously presented in $[36,37]$. The model, presented here, is enhanced from previous work, including the inclusion of stroke plane dynamics, calculation of the aerodynamic forces and moments based on a quasi-steady/blade-element method, and a more accurate accounting of the angular velocity of the wings. Additionally, the model will be presented in comparison with the standard aircraft model. The main equation is

$$
\sum_{i=1}^{n}\left[\dot{\overline{\mathbf{p}}}_{i} \cdot \bar{\gamma}_{\mathbf{i j}}+\left(\dot{\overline{\mathbf{H}}}_{i}+m_{i} \bar{\rho}_{\mathbf{c i}} \times \dot{\overline{\mathbf{v}}}_{i}\right) \cdot \bar{\beta}_{\mathbf{i j}}\right]=Q_{j}
$$

where $i$ denotes the number of rigid bodies and $j$ denotes the number of generalized coordinates (with associated quasi velocities). In Eq. (2), linear momentum is defined as $\overline{\mathbf{p}}_{i}$ and angular momentum is

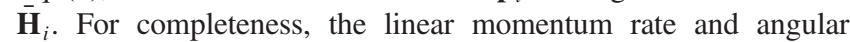
momentum rate of the $i$ th rigid body are defined as

$$
\dot{\overline{\mathbf{p}}}_{i}=m_{i}\left(\dot{\overline{\mathbf{v}}}+\dot{\bar{\rho}}_{c i}\right) \quad \text { and } \quad \dot{\overline{\mathbf{H}}}_{i}=\mathbf{I}_{\mathbf{i}} \cdot \dot{\bar{\omega}}_{\mathbf{i}}+\bar{\omega}_{\mathbf{i}} \times \mathbf{I}_{\mathbf{i}} \cdot \bar{\omega}_{\mathbf{i}}
$$

The method has a few main advantages. One, since the Principle of Virtual Work is used to calculate the forces and moments for each generalized coordinate, the constraint forces between the wing and the central body are neglected since constraints forces do not perform virtual work. Two, the method allows the choice of reference points for the velocity of each body. The wings are assumed to be attached to the central body by joints that allow three degrees of freedom. To simplify the derivation, and eliminate the need for tracking the absolute velocity and acceleration of the wings in an inertial frame, the velocity reference points for the wings are chosen to be the respective wing joints. Finally, the inertia tensor for the individual bodies is calculated with respect to the reference point and does not need to be calculated at the time-varying center of mass of the system.

\section{B. Reference Frames}

To accurately describe the motion of the body with respect to an inertial frame, and the motion of the wings with respect to the body, six reference frames are required. The first reference frame is an inertial frame. The absolute velocity and position of the FWMAV is described with respect to the inertial frame. The $B$ frame is a bodyfixed frame attached to the central body of the FWMAV with origin at the center of mass of the body and is depicted in Fig. 1a. The frame is oriented with positive $x$ axis along the longitudinal axis of the central body. The $y$ axis is perpendicular to the $x$ axis and is positive out of the right side of the vehicle. The $z$ axis is positive downward and perpendicular to the $x-y$ plane. The unit vectors of the $B$ frame are denoted by $\hat{b}_{x}, \hat{b}_{y}$, and $\hat{b}_{z}$. An $x$-z plane of mass symmetry is assumed for the central body. In addition to the $B$ frame, the stroke plane frames are two body-fixed frames originated at the wing joints. The stroke plane frames are denoted by $R_{\mathrm{sp}}$ and $L_{\mathrm{sp}}$ and have initial 


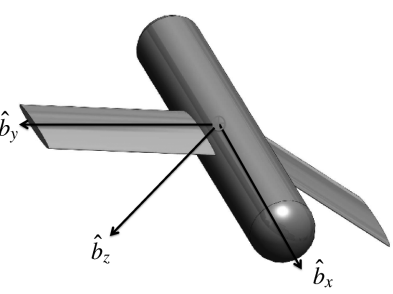

a) $B$ frame

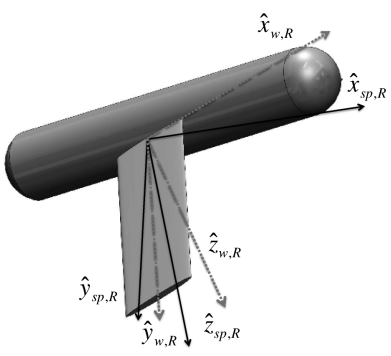

b) Right stroke plane and wing frames

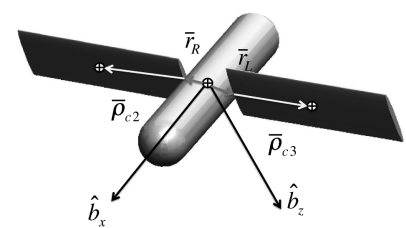

c) Reference vectors

Fig. 1 Model representation with reference frames and reference vectors.

orientation parallel to the $B$ frame. The orientation is rotated by an angle $\beta$ about the $\hat{b}_{y}$ axis of the $B$ frame to the stroke plane. The stroke plane defines the mean motion of the wing. The stroke plane angle defines the orientation of the stroke plane relative to the longitudinal axis of the central body. The $y$ axis of the stroke plane frames will always remain parallel to the $B$ frame. The $x$ and $z$ axes of $R_{\text {sp }}$ and $L_{\text {sp }}$ will be rotated by the fixed angles $\beta_{R}$ and $\beta_{L}$. The last two frames are fixed frames attached to the wings. The initial orientation of the wing frames is parallel to the stroke plane frames with an origin coincident with the wing joint. The wing frames, $R_{w}$ and $L_{w}$, move with the rotation of the wings and enable the calculation of the wings' orientations with respect to the stroke plane, and by extension, the central body. The right stroke plane frame and wing frame are depicted in Fig. 1b. The stroke plane frame is represented by solid lines and unit vectors with the subscript $\mathrm{sp}, R$. The wing frame is represented by dashed lines and unit vectors with the subscript $w, R$.

\section{Orientation}

The orientation of the central body is determined by 3-2-1 Euler angles with respect to the inertial (fixed) frame. The nomenclature for the angles is consistent with the NASA standard for aircraft. The orientation of the stroke plane with respect to the body is denoted by the angles $\beta_{R}$ and $\beta_{L}$ and is fixed for a given flight condition. The orientation of the wings with respect to the stroke plane is determined by the deviation, pitch, and flap angles of the wings. The wing angles are $\delta, \alpha$, and $\zeta$. The stroke plane angle and angle of attack are shown in Fig. 2a. A common, although not completely accepted, nomenclature choice for the deviation (elevation) and flap (sweep) angles are $\theta$ and $\phi$, respectively. Here, $\delta$ is chosen for the deviation angle and $\zeta$ is chosen for the sweep angle to avoid confusion with the pitch and roll angles of the central body. The relation of the $\delta$ and $\zeta$ angles to the central body are shown in Figs. $2 \mathrm{~b}$ and $2 \mathrm{c}$.

Positive rotations are consistent with the right hand rule. A positive angle of attack is "up" in the stroke plane frame. A positive deviation (elevation) angle is down and a positive flap (sweep) angle is forward. The kinematics of the wings, usually sinusoidal functions to be discussed later, will always be set so that positive motion is "forward" and "down."

\section{Rotation Matrices}

The rotation matrix from the inertial frame to the body frame, $\mathbf{R}_{B}$, is determined by proper combination of the 3-2-1 Euler angles. The standard combination can be found in $[1, \underline{35}]$. The rotation matrix from the body to the stroke plane frame is

$$
\mathbf{R}_{\beta}=\left[\begin{array}{ccc}
\cos \beta & 0 & -\sin \beta \\
0 & 1 & 0 \\
\sin \beta & 0 & \cos \beta
\end{array}\right]
$$

where $\beta_{R}$ and $\beta_{L}$ are substituted for the proper stroke plane frame. The orientation for the right and left wings with respect to the $R_{\mathrm{sp}}$ and $L_{\mathrm{sp}}$ is determined by the 3-1-2 Euler angles, where $\zeta=3, \alpha=2$, and $\delta=1$. The choice of 3-1-2 Euler angles matches the requirements of the system in a physical sense. For example, the radial position of a point on the wing can be tracked in the stroke plane frame using spherical coordinates with the angles $\zeta$ and $\delta$. The choice of 3-1-2 Euler angles gives spherical coordinates for a radial $(y)$ position of the wing when transformed from the wing frame to the stroke plane frame. The singularity for 3-1-2 Euler angles, using the chosen nomenclature, is at $\delta= \pm(\pi / 2)$ and will not be reached by the wing stroke kinematics. The rotation matrices for the right wing are

$$
\begin{aligned}
& \mathbf{R}_{\delta_{R}}= {\left[\begin{array}{ccc}
1 & 0 & 0 \\
0 & \cos \delta_{R} & \sin \delta_{R} \\
0 & -\sin \delta_{R} & \cos \delta_{R}
\end{array}\right], } \\
& \mathbf{R}_{\alpha_{R}}= {\left[\begin{array}{ccc}
\cos \alpha_{R} & 0 & -\sin \alpha_{R} \\
0 & 1 & 0 \\
\sin \alpha_{R} & 0 & \cos \alpha_{R}
\end{array}\right], \quad \text { and } } \\
& \mathbf{R}_{\zeta_{R}}=\left[\begin{array}{ccc}
\cos \zeta_{R} & -\sin \zeta_{R} & 0 \\
\sin \zeta_{R} & \cos \zeta_{R} & 0 \\
0 & 0 & 1
\end{array}\right]
\end{aligned}
$$

The rotation matrices are combined according to

$$
\mathbf{R}_{R}=\mathbf{R}_{\alpha_{R}} \mathbf{R}_{\delta_{R}} \mathbf{R}_{\zeta_{R}}
$$

The rotation matrices for the left wing, with respect to the left stroke plane, are combined in the same manner as in Eq. (6). The

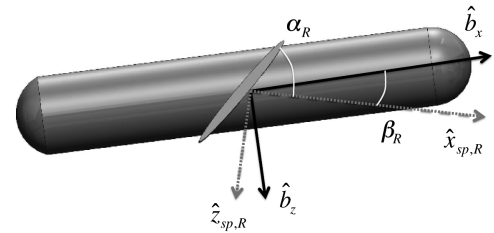

a) $\alpha_{R}$ and $\beta_{R}$

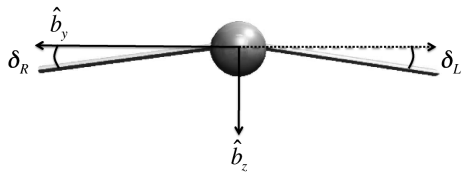

b) $\delta_{R}$ and $\delta_{L}$

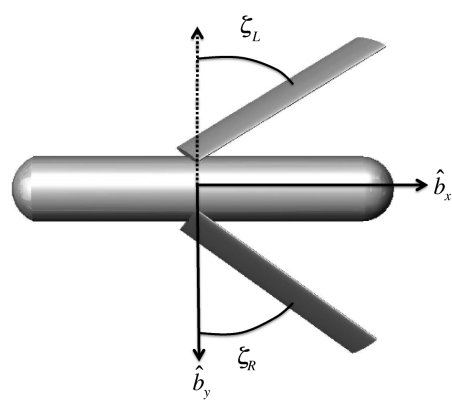

c) $\zeta_{R}$ and $\zeta_{L}$

Fig. 2 Wing angles and stroke plane angles. 
negative signs for the rotation matrices for the $\zeta$ and $\delta$ angles are interchanged for the right and left wings. The sign change is due to the fact that "positive" motion of the wings is forward, which is a positive rotation for the left wing by the angle $\zeta_{L}$, but a negative rotation for the right wing by the angle $\zeta_{R}$. Likewise, positive downward motion is a positive rotation of the angle $\delta_{R}$ for the right wing, but a negative rotation by the angle $\delta_{L}$ for the left wing. The correct sign ensures proper cancellation of forces and moments in the $B$ frame when the flapping is symmetrical, which will be discussed in Sec. III.G.

\section{Generalized Coordinates}

A FWMAV truly only has six degrees of freedom: the three translational and rotational degrees of freedom of the central body. However, each of the wings has three holonomically constrained degrees of freedom relative to the central body. Combining the six true degrees of freedom and six holonomically constrained degrees of freedom, we can view the system as having 12 independent degrees of freedom $[22,23,34]$. As a result, we need 12 generalized coordinates to accurately describe the system. The inertial position is described by $X, Y$, and $Z$. The orientation of the body with respect to an inertial frame is determined by the angles $\psi, \theta$, and $\phi$. The orientation of the right wing is described by the angles $\delta_{R}, \alpha_{R}$, and $\zeta_{R}$. The orientation of the left wing is described by the angles $\delta_{L}, \alpha_{L}$, and $\zeta_{L}$. Sum total, the 12 degrees of freedom for the system are described by the generalized coordinates, $\mathbf{q}_{j}$, listed together as

$$
\mathbf{q}_{j}=\left[\begin{array}{llllllllllll}
X & Y & Z & \psi & \theta & \phi & \delta_{R} & \alpha_{R} & \zeta_{R} & \delta_{L} & \alpha_{L} & \zeta_{L}
\end{array}\right]
$$

The associated quasi velocities of the system, $\mathbf{u}_{j}$, are

$\mathbf{u}_{j}=\left[\begin{array}{llllllllllll}u & v & w & p & q & r & p_{\mathrm{RW}} & q_{\mathrm{RW}} & r_{\mathrm{RW}} & p_{\mathrm{LW}} & q_{\mathrm{LW}} & r_{\mathrm{LW}}\end{array}\right]$

The variables $u, v$, and $w$ described the translational velocity of the central body in the $B$ frame. The variables $p, q$, and $r$ describe the angular velocity of the central body in the $B$ frame. The final six quasi velocities are the angular velocity components of the wings in the stroke plane and expressed in the $B$ frame.

\section{Reference Vectors}

The reference vectors are denoted by $\bar{\rho}_{c i}$ in Eq. (2). For each body, a reference point is chosen. The reference vectors denote the position of the center of mass of the $i$ th body with respect to the reference point. For the central body, the reference point is chosen to be its respective center of mass. As a direct result, the reference vector $\bar{\rho}_{c 1}$ is identically zero. The reference points for each of the wings are chosen to be the respective wing joints. We assume that each wing is rigid. The vectors describing the position of the wing center of mass relative to the wing joint, in the wing frame, are $\bar{\rho}_{c 2, w}$ and $\bar{\rho}_{c 3, w}$. To express the reference vectors in the body frame, the vectors are transformed from the wing frame according to

$$
\bar{\rho}_{c 2}=\mathbf{R}_{\beta_{R}}^{T} \mathbf{R}_{R}^{T} \bar{\rho}_{c 2, w} \quad \text { and } \quad \bar{\rho}_{c 3}=\mathbf{R}_{\beta_{L}}^{T} \mathbf{R}_{L}^{T} \bar{\rho}_{c 3, w}
$$

The required accelerations of the reference vectors are denoted by $\ddot{\bar{\rho}}_{c i}$. The acceleration of the reference vector for the central body is identically zero. The acceleration vectors for the right and left wings' reference vectors are obtained according to the following derivation. The reference vectors are defined according to Eq. (9). The velocity of the reference vectors is obtained from

$$
\dot{\bar{\rho}}_{c 2}=\frac{\partial}{\partial t}\left(\mathbf{R}_{\beta_{R}}^{T} \mathbf{R}_{R}^{T} \bar{\rho}_{c 2, w}\right)+\bar{\omega}_{2} \times \bar{\rho}_{c 2}
$$

The angular velocities of the wings in the $B$ frame and are obtained according to Eq. (20), with appropriate substitutions made for the left wing. The time derivative of the right wing reference vector is

$$
\frac{\partial}{\partial t}\left(\mathbf{R}_{\beta_{R}}^{T} \mathbf{R}_{R}^{T} \bar{\rho}_{c 2, w}\right)=\mathbf{R}_{\beta_{R}}^{T}\left(\dot{\mathbf{R}}_{R}^{T} \bar{\rho}_{c 2, w}+\mathbf{R}_{R}^{T} \dot{\bar{\rho}}_{c 2, w}\right)
$$

where the stroke plane angle, $\beta_{R}$, is assumed to be constant and the derivative of the reference vector, in the wing frame, is zero. The result is

$$
\frac{\partial}{\partial t}\left(\mathbf{R}_{\beta_{R}}^{T} \mathbf{R}_{R}^{T} \bar{\rho}_{c 2, w}\right)=\mathbf{R}_{\beta_{R}}^{T}\left(\dot{\mathbf{R}}_{R}^{T} \bar{\rho}_{c 2, w}\right)
$$

The time derivative of the transpose of the rotation matrix, $\dot{\mathbf{R}}_{R}^{T}$, is obtained from the relationship between angular velocity and rotation matrices. The time derivative of the transpose of the rotation matrix is equal to

$$
\dot{\mathbf{R}}_{R}^{T}=\mathbf{R}_{R}^{T} \tilde{\omega}_{2, \mathrm{sp}}
$$

where $\tilde{\omega}_{2, \text { sp }}$ denotes the skew-symmetric (or cross) matrix of the angular velocity of the right wing with respect to the stroke plane frame, which will be defined in Eq. (19). A similar procedure is used to derive the acceleration of the left wing reference vector. The acceleration of the reference vectors, with respect to the inertial frame and expressed in the $B$ frame, is calculated according to

$$
\ddot{\bar{\rho}}_{c 2}=\frac{\partial}{\partial t} \dot{\bar{\rho}}_{c 2}+\dot{\bar{\omega}}_{2} \times \bar{\rho}_{c 2}+\bar{\omega}_{2} \times\left(\bar{\omega}_{2} \times \bar{\rho}_{c 2}\right)
$$

Additionally, vectors are defined from the center of mass of the body frame to the wing joints. Since the central body is assumed to be rigid, then the vectors are fixed and their respective components are constant. The vector from the origin of the $B$ frame to the right wing joint is $\bar{r}_{R}$ and to the left wing joint is $\bar{r}_{L}$. The wing reference vectors and joint reference vectors are depicted in Fig. 1c. Depending on the configuration of the flapping wing aircraft, $\bar{r}_{R}$ and $\bar{r}_{L}$ may, or may not, have components in all directions in the $B$ frame. The components of the vectors $\bar{r}_{R}$ and $\bar{r}_{L}$ are defined by $R_{x}, R_{y}, R_{z}, L_{x}, L_{y}$, and $L_{z}$.

\section{E. Velocities}

The velocities of each of the bodies are now defined. The translational velocity of the central body, expressed in the $B$ frame and with respect to the inertial frame, is

$$
\overline{\mathbf{v}}_{1}=u \hat{b}_{x}+v \hat{b}_{y}+w \hat{b}_{z}
$$

The angular velocity of the central body, expressed in the $B$ frame and with respect to the inertial frame, is

$$
\bar{\omega}_{1}=p \hat{b}_{x}+q \hat{b}_{y}+r \hat{b}_{z}
$$

Since the wing joints are chosen to be the reference points, the reference velocity, for each of the wings, is the velocity of the respective wing joint in the $B$ frame. The velocities of the right and left wing joints are

$$
\overline{\mathbf{v}}_{2}=\overline{\mathbf{v}}_{1}+\bar{\omega}_{1} \times \overline{\mathbf{r}}_{R} \quad \text { and } \quad \overline{\mathbf{v}}_{3}=\overline{\mathbf{v}}_{1}+\bar{\omega}_{1} \times \overline{\mathbf{r}}_{L}
$$

The angular velocities of the wings are a function of the wing angles and angular rates. The angular velocities of the wings, with respect to the stroke plane, are calculated according to the 3-1-2 Euler angle relationship. The angular velocity of the right wing is calculated according to

$$
\bar{\omega}_{2, \mathrm{sp}}=\mathbf{R}_{R}\left[\begin{array}{c}
0 \\
0 \\
-\dot{\zeta}_{R}
\end{array}\right]+\mathbf{R}_{\alpha_{R}} \mathbf{R}_{\delta_{R}}\left[\begin{array}{c}
\dot{\delta}_{R} \\
0 \\
0
\end{array}\right]+\mathbf{R}_{\alpha_{R}}\left[\begin{array}{c}
0 \\
\dot{\alpha}_{R} \\
0
\end{array}\right]
$$

In component form, the angular velocity of the right wing with respect to the stroke plane, is

$$
\bar{\omega}_{2, \mathrm{sp}}=\left[\begin{array}{c}
p_{\mathrm{RW}} \\
q_{\mathrm{RW}} \\
r_{\mathrm{RW}}
\end{array}\right]=\left[\begin{array}{c}
\left(\cos \alpha_{R}\right) \dot{\delta}_{R}+\left(\sin \alpha_{R} \cos \delta_{R}\right) \dot{\zeta}_{R} \\
\dot{\alpha}_{R}-\left(\sin \delta_{R}\right) \dot{\zeta}_{R} \\
\left(\sin \alpha_{R}\right) \dot{\delta}_{R}-\left(\cos \alpha_{R} \cos \delta_{R}\right) \dot{\zeta}_{R}
\end{array}\right]
$$


The total angular velocity of the right wing with respect to the inertial frame, and expressed in the $B$ frame, is

$$
\bar{\omega}_{2}=\bar{\omega}_{1}+\mathbf{R}_{\beta_{R}}^{T} \bar{\omega}_{2, \mathrm{sp}}
$$

A similar procedure is used to develop the angular velocity of the left wing. The total angular velocity of the right wing, expressed in Eq. (20), is the angular velocity of the wing expressed in the body frame but with respect to the inertial frame. It is important to note, consistent with the development of the orientation of the wings with respect to the stroke planes and the right hand rule, that the signs are opposite for $\dot{\delta}_{R}$ and $\dot{\delta}_{L}$ and $\dot{\zeta}_{R}$ and $\dot{\zeta}_{L}$, which results (for symmetric flapping) in $-p_{\mathrm{LW}}=p_{\mathrm{RW}}$ and $r_{\mathrm{LW}}=-r_{\mathrm{RW}}$ in the $B$ frame.

\section{F. Velocity and Angular Velocity Coefficients}

Velocity and angular velocity coefficients arise from the calculation of virtual work performed by forces and moments [34]. Each velocity and angular velocity coefficient is a vector. The velocity and angular velocity coefficients are calculated according to

$$
\bar{\gamma}_{i j}=\frac{\partial \overline{\mathbf{v}}_{i}}{\partial \mathbf{u}_{j}} \quad \text { and } \quad \bar{\beta}_{i j}=\frac{\partial \bar{\omega}_{i}}{\partial \mathbf{u}_{j}}
$$

The velocities and angular velocities for the $i$ th rigid body are defined in Sec. III.E. The system quasi velocities, $\mathbf{u}_{j}$, are detailed in Sec. III.C. Since each coefficient is a vector, the coefficients are combined with the other elements of the equations generated by Eq. (2) according to inner product rules. With 12 coordinates and three rigid bodies, the total number of velocity coefficients and angular velocity coefficients is 36 each. The velocity coefficients for all three rigid bodies, due to the translational motion of the central body, can be summarized as

$$
\left[\begin{array}{l}
\bar{\gamma}_{11} \\
\bar{\gamma}_{12} \\
\bar{\gamma}_{13}
\end{array}\right]=\left[\begin{array}{l}
\bar{\gamma}_{21} \\
\bar{\gamma}_{22} \\
\bar{\gamma}_{23}
\end{array}\right]=\left[\begin{array}{l}
\bar{\gamma}_{31} \\
\bar{\gamma}_{32} \\
\bar{\gamma}_{33}
\end{array}\right]=\mathbb{I}^{3 \times 3}\left[\begin{array}{l}
\hat{b}_{x} \\
\hat{b}_{y} \\
\hat{b}_{z}
\end{array}\right]
$$

The velocity coefficients of the right wing and left wings, due to the angular velocity of the central body are

$$
\begin{gathered}
{\left[\begin{array}{l}
\bar{\gamma}_{24} \\
\bar{\gamma}_{25} \\
\bar{\gamma}_{26}
\end{array}\right]=\left[\begin{array}{ccc}
0 & -R_{z} & R_{y} \\
R_{z} & 0 & -R_{x} \\
-R_{y} & R_{x} & 0
\end{array}\right]\left[\begin{array}{l}
\hat{b}_{x} \\
\hat{b}_{y} \\
\hat{b}_{z}
\end{array}\right] \text { and }} \\
{\left[\begin{array}{l}
\bar{\gamma}_{34} \\
\bar{\gamma}_{35} \\
\bar{\gamma}_{36}
\end{array}\right]=\left[\begin{array}{ccc}
0 & -L_{z} & L_{y} \\
L_{z} & 0 & -L_{x} \\
-L_{y} & L_{x} & 0
\end{array}\right]\left[\begin{array}{l}
\hat{b}_{x} \\
\hat{b}_{y} \\
\hat{b}_{z}
\end{array}\right]}
\end{gathered}
$$

The velocity coefficients due to the angular velocity components of the wings are all identically zero, for each rigid body. The nonzero angular velocity coefficients for the central body and the wings are

$$
\left[\begin{array}{l}
\bar{\beta}_{14} \\
\bar{\beta}_{15} \\
\bar{\beta}_{16}
\end{array}\right]=\left[\begin{array}{l}
\bar{\beta}_{24} \\
\bar{\beta}_{25} \\
\bar{\beta}_{26}
\end{array}\right]=\left[\begin{array}{l}
\bar{\beta}_{34} \\
\bar{\beta}_{35} \\
\bar{\beta}_{36}
\end{array}\right]=\mathbb{I}^{3 \times 3}\left[\begin{array}{l}
\hat{b}_{x} \\
\hat{b}_{y} \\
\hat{b}_{z}
\end{array}\right]
$$

The angular velocity coefficients for the central body due to the angular velocity of the wings and translational velocity of the central body are identically zero. Since the total angular velocity of the wings is a combination of the angular velocity of the central body and the angular velocity of the wings with respect to the body frame, the angular velocity coefficients from the wings due to the angular velocity of the central body are identical. The angular velocity coefficients for the right wing, due to the angular velocity of the right wing, and the angular velocity coefficients of the left wing, due to the angular velocity of the left wing, are

$$
\left[\begin{array}{c}
\bar{\beta}_{2,7} \\
\bar{\beta}_{2,8} \\
\bar{\beta}_{2,9}
\end{array}\right]=\mathbf{R}_{\beta_{R}}^{T}\left[\begin{array}{c}
\hat{b}_{x} \\
\hat{b}_{y} \\
\hat{b}_{z}
\end{array}\right] \text { and }\left[\begin{array}{c}
\bar{\beta}_{3,10} \\
\bar{\beta}_{3,11} \\
\bar{\beta}_{3,12}
\end{array}\right]=\mathbf{R}_{\beta_{L}}^{T}\left[\begin{array}{c}
\hat{b}_{x} \\
\hat{b}_{y} \\
\hat{b}_{z}
\end{array}\right]
$$

The angular velocity coefficients of the right wing, due to the angular velocity of the left wing, are identically zero. The same is true for the angular velocity coefficients of the left wing due to the angular velocity of the right wing.

\section{G. Forces}

The forces are calculated according to the principle of virtual work. The principle of virtual work calculates the generalized forces and moments acting on the system due to an arbitrary virtual displacement. The derivation of the principle of virtual work is detailed in [33-35]. The generalized forces and moments are calculated according to

$$
Q_{j}=\sum_{i=1}^{n}\left(\mathbf{F}_{i} \cdot \bar{\gamma}_{i j}+\mathbf{M}_{i} \cdot \bar{\beta}_{i j}\right)
$$

for each $j$ th coordinate. The resultant forces, $\mathbf{F}_{i}$, and moments, $\mathbf{M}_{i}$ are determined for each rigid body. The resultant forces acting on the central body are due to the acceleration due to gravity and the aerodynamic forces generated on the central body due to its translation. There are zero resultant moments acting directly on the central body. The resultant forces acting on the wings are the aerodynamic forces generated by the motion of the wings, acting at the wing center of pressure, and the gravity force, acting at the center of mass of the wing. The resultant moments on the wings are calculated with respect to the wing joints and include contributions from the aerodynamic forces, the gravity force, and the control moments applied to obtain the desired wing motion. The generalized forces affecting the translation of the central body are

$$
\left[\begin{array}{l}
Q_{1} \\
Q_{2} \\
Q_{3}
\end{array}\right]=\left[\begin{array}{l}
F_{x} \\
F_{y} \\
F_{z}
\end{array}\right]+\left(m_{1}+m_{2}+m_{3}\right) \mathbf{R}_{B}\left[\begin{array}{l}
0 \\
0 \\
g
\end{array}\right]
$$

The generalized forces affecting the rotation of the central body are

$$
\left[\begin{array}{l}
Q_{4} \\
Q_{5} \\
Q_{6}
\end{array}\right]=\bar{M}_{\text {aero }}+\bar{M}_{g}
$$

where $\bar{M}_{\text {aero }}$ is the vector of aerodynamic moments acting on the central body and $\bar{M}_{g}$ is the moments due to gravity of the wings on the central body. The moments due to gravity are calculated according to

$$
\bar{M}_{g}=\bar{M}_{g, R}+\bar{M}_{g, L}
$$

where the moments due to gravity for the right wing and left wing are calculated according to

$$
\begin{gathered}
\bar{M}_{g, R}=\left(\bar{r}_{R}+\bar{\rho}_{c 2}\right) \times\left(m_{2}\right) \mathbf{R}_{B}\left[\begin{array}{l}
0 \\
0 \\
g
\end{array}\right] \text { and } \\
\bar{M}_{g, L}=\left(\bar{r}_{L}+\bar{\rho}_{c 3}\right) \times\left(m_{3}\right) \mathbf{R}_{B}\left[\begin{array}{l}
0 \\
0 \\
g
\end{array}\right]
\end{gathered}
$$

The generalized forces $Q_{7}, Q_{8}$, and $Q_{9}$ are the control moments for the right wing. $Q_{10}, Q_{11}, Q_{12}$ are the control moments for the left wing. $Q_{8}$ and $Q_{11}$ control the angle of attack of the wings, right and left wing, respectively. $Q_{7}$ and $Q_{10}$ control the deviation angle of the wings, while $Q_{9}$ and $Q_{12}$ control the flap angle of the wings. The forces and moments produced by the wings will be the result of aerodynamic modeling of the wing. For the moment, we will assume the motion will produce a force normal, $F_{N}$, and tangential, $F_{T}$, to the wing. The forces will be calculated in the wing frame and 
transformed to the stroke plane and body frame. We define the total aerodynamic forces acting on the body, expressed in the $B$ frame as

$$
\bar{F}_{\text {aero }}=F_{x} \hat{b}_{x}+F_{y} \hat{b}_{y}+F_{z} \hat{b}_{z}
$$

The total aerodynamic moments are defined as

$$
\bar{M}_{\text {aero }}=L \hat{b}_{x}+M \hat{b}_{y}+N \hat{b}_{z}
$$

Assuming a normal and tangential force produced by each wing, the forces expressed in the body frame are calculated according to

$$
\left[\begin{array}{c}
F_{x, R} \\
F_{y, R} \\
F_{z, R}
\end{array}\right]=\mathbf{R}_{\beta_{R}}^{T} \mathbf{R}_{R}^{T}\left[\begin{array}{c}
F_{T, R} \\
0 \\
F_{N, R}
\end{array}\right] \text { and }\left[\begin{array}{c}
F_{x, L} \\
F_{y, L} \\
F_{z, L}
\end{array}\right]=\mathbf{R}_{\beta_{L}}^{T} \mathbf{R}_{L}^{T}\left[\begin{array}{c}
F_{T, L} \\
0 \\
F_{N, L}
\end{array}\right]
$$

If the chosen aerodynamic model produces identical normal and tangential forces for the right and left wings, then in the body frame the $F_{x}$ and $F_{z}$ forces are the same and the $F_{y}$ forces will perfectly cancel each other out, when the flapping is symmetrical with respect to the central body. A rotation from the stroke plane will not change the $F_{y}$ forces and will only change the magnitude/combination of the $F_{x}$ and $F_{z}$ forces. The aerodynamic centers of pressure of the wings are calculated based on the morphology of the wings. The $x$ - and $y$ positions of the aerodynamic centers of pressure, in the wing frame, are obtained with the assistance of the following equations:

$$
\hat{r}_{2}=\sqrt{\frac{\int_{0}^{R} c(r) r^{2} \mathrm{~d} r}{R^{2} A_{w}}} \text { and } \hat{c}=\frac{\int_{0}^{R} c^{2}(r) r \mathrm{~d} r}{\hat{r}_{2} R A_{w} c_{\max }}
$$

which are based on the geometry of the wings $[20,21]$. The resulting aerodynamic center is at the following coordinates in the wing frames:

$$
\bar{\rho}_{\mathrm{ac}, R, w}=\left[\begin{array}{c}
\frac{c_{R}}{4} \\
\hat{r}_{2, R} b_{R} \\
0
\end{array}\right] \quad \text { and } \quad \bar{\rho}_{\mathrm{ac}, L, w}=\left[\begin{array}{c}
\frac{c_{L}}{4} \\
-\hat{r}_{2, L} b_{L} \\
0
\end{array}\right]
$$

In Eq. (35), $c_{R}$ and $c_{L}$ are the chords of the respective wings, $b_{R}$ and $b_{L}$ are the semispans of the wings and $\hat{r}_{2}$ denotes the normalized aerodynamic center of pressure. The aerodynamic centers are transformed into the $B$ frame in the same manner as the forces are transformed in Eq. (33). The total aerodynamic moment in the $B$ frame, for each wing, is given by

$$
\begin{gathered}
\bar{M}_{\mathrm{aero}, R}=\left(\bar{r}_{R}+\bar{\rho}_{\mathrm{ac}, R}\right) \times \bar{F}_{\mathrm{aero}, R} \text { and } \\
\bar{M}_{\mathrm{aero}, L}=\left(\bar{r}_{L}+\bar{\rho}_{\mathrm{ac}, L}\right) \times \bar{F}_{\mathrm{aero}, L}
\end{gathered}
$$

In component form, the total aerodynamic moments in the $B$ frame are

$$
\left[\begin{array}{c}
L \\
M \\
N
\end{array}\right]=\left[\begin{array}{l}
\rho_{\mathrm{RW}, y} F_{z, R}-\rho_{\mathrm{RW}, z} F_{y, R}+\rho_{\mathrm{LW}, y} F_{z, L}-\rho_{\mathrm{LW}, z} F_{y, L} \\
\rho_{\mathrm{RW}, z} F_{x, R}-\rho_{\mathrm{RW}, x} F_{z, R}+\rho_{\mathrm{LW}, z} F_{x, L}-\rho_{\mathrm{LW}, x} F_{z, L} \\
\rho_{\mathrm{RW}, y} F_{x, R}-\rho_{\mathrm{RW}, x} F_{y, R}+\rho_{\mathrm{LW}, y} F_{x, L}-\rho_{\mathrm{LW}, x} F_{y, L}
\end{array}\right]
$$

where $\bar{\rho}_{\mathrm{RW}}=\bar{r}_{R}+\bar{\rho}_{\mathrm{ac}, R}$ and $\bar{\rho}_{\mathrm{LW}}=\bar{r}_{L}+\bar{\rho}_{\mathrm{ac}, L}$. For symmetrical flapping, we showed previously that $F_{y, R}=-F_{y, L}$. Similarly, under the constraint of symmetrical flapping, $\rho_{\mathrm{ac}, R, y}=-\rho_{\mathrm{ac}, L, y}$. Good engineering design will place the $y$ components of $\bar{r}_{R}$ and $\bar{r}_{L}$ equal and opposite, or asymmetric moments result. Therefore, if the flapping is symmetric and the stroke plane angles are equal, we can perform proper cancellations and obtain

$$
\left[\begin{array}{c}
L \\
M \\
N
\end{array}\right]=\left[\begin{array}{c}
0 \\
\rho_{\mathrm{RW}, z} F_{x, R}-\rho_{\mathrm{RW}, x} F_{z, R}+\rho_{\mathrm{LW}, z} F_{x, L}-\rho_{\mathrm{LW}, x} F_{z, L} \\
0
\end{array}\right]
$$

which is expected if the flapping is perfectly symmetrical, the normal and tangential forces are identical for both wings when symmetrical flapping occurs, and the wings have the same morphological parameters.

\section{H. Equations of Motion}

The equations of motion are now summarized for each of the generalized coordinates. The equations of motion governing the translation of the central body are

$$
\mathbb{I}^{3 \times 3}\left(\sum_{i=1}^{3}\left(m_{i}+\ddot{\bar{\rho}}_{c i}\right)\right)=\left[\begin{array}{lll}
Q_{1} & Q_{2} & Q_{3}
\end{array}\right]^{T}
$$

The equations of motion governing the rotation of the central body are

$$
\begin{aligned}
& m_{2} \bar{r}_{R} \times\left(\dot{\overline{\mathbf{v}}}_{2}+\ddot{\bar{\rho}}_{c 2}\right)+m_{3} \bar{r}_{L} \times\left(\dot{\overline{\mathbf{v}}}_{3}+\ddot{\bar{\rho}}_{c 3}\right)+\sum_{i=1}^{3}\left(\dot{\overline{\mathbf{H}}}_{i}+m_{i} \bar{\rho}_{\mathbf{c i}} \times \dot{\overline{\mathbf{v}}}_{i}\right) \\
& \quad=\left[\begin{array}{lll}
Q_{4} & Q_{5} & Q_{6}
\end{array}\right]^{T}
\end{aligned}
$$

The equations of motion governing the motion of the right wing are

$$
\mathbf{R}_{\beta_{R}}^{T}\left(\mathbf{I}_{2} \dot{\bar{\omega}}_{2}+\bar{\omega}_{2} \times \mathbf{I}_{2} \bar{\omega}_{2}+m_{2} \bar{\rho}_{c 2} \times \dot{\overline{\mathbf{v}}}_{2}\right)=\left[\begin{array}{lll}
Q_{7} & Q_{8} & Q_{9}
\end{array}\right]^{T}
$$

The equations of motion governing the motion of the left wing are

$$
\mathbf{R}_{\beta_{L}}^{T}\left(\mathbf{I}_{3} \dot{\bar{\omega}}_{3}+\bar{\omega}_{3} \times \mathbf{I}_{3} \bar{\omega}_{3}+m_{3} \bar{\rho}_{c 3} \times \dot{\overline{\mathbf{v}}}_{3}\right)=\left[\begin{array}{lll}
Q_{10} & Q_{11} & Q_{12}
\end{array}\right]^{T}
$$

\section{Wingless Model}

We previously presented the standard aircraft model in Eq. (1). From the derivation of the full nonlinear equations previously presented, we can make the following substitutions into Eq. (1): $\bar{\omega}_{1}=\bar{\omega}_{b}, \mathbf{I}_{1}=\mathbf{I}_{b}$, and $\overline{\mathbf{v}}_{1}=\overline{\mathbf{v}}_{b}$. The mass of the body will equal the entire mass of the system, both central body and wings. The inertia tensor will be calculated based on the mass of the system, adding the mass of the wings to that of the central body. Additionally, the aerodynamic forces and moments generated by the wings will be calculated in the same manner as previously presented. The nonlinear model derived in this paper will be simulated against the standard aircraft model. Furthermore, the standard model and the derived model will be simulated against a slightly modified standard aircraft model. The standard aircraft model will be modified according to

$$
\begin{aligned}
& \dot{\overline{\mathbf{v}}}_{1}=\frac{1}{m_{1}}\left(\bar{W}_{1}+\bar{F}_{\text {aero }}\right)+\bar{\omega}_{b} \times \overline{\mathbf{v}}_{b} \\
& \dot{\bar{\omega}}_{b}=\mathbf{I}_{b}^{-1}\left(\bar{\omega}_{b} \times \mathbf{I}_{b} \bar{\omega}_{b}+\bar{M}_{\text {aero }}+\bar{M}_{g}\right)
\end{aligned}
$$

where $\bar{M}_{g}$ is the gravity moment acting on the central body center of mass due to the motion of the wings' center of mass. The gravity moment calculation is presented in Eqs. (29) and (30).

\section{Simulation}

\section{A. Body Parameters}

The FWMAV is modeled after a hawkmoth as presented in [3] . The specific specimen chosen is F1. A hawkmoth is chosen because, according to Willmott and Ellington in [3], the wing strokes can be considered the most "representative" of insect wing strokes. Additionally, the flapping frequency for hawkmoths is generally between 24 and $26 \mathrm{~Hz}$, which can be replicated by current technology (as opposed to using a bumblebee or fruitfly, where the flapping frequency is on the order of 150 and $200 \mathrm{~Hz}$, respectively). The total mass of the FWMAV will be set at $1648 \mathrm{mg}$, with the wings accounting for $5.7 \%$ of the total body mass.

\section{Central Body}

The central body is modeled as a cylinder with a constant radius. The mass of the body is set at $1554 \mathrm{mg}$, with a length of $42.1 \mathrm{~mm}$, and a constant radius of $6 \mathrm{~mm}$. The parameters are similar to those used in 
[38]. The length is calculated based off of the $\hat{L}$ parameter in [3]. The radius is calculated from the $\hat{l}_{1}$ parameter in [3]. Since the body is modeled as a cylinder, the inertia tensor for the central body will be diagonal. The wings are assumed to be mounted at wing joints with components such that $R_{y}=r_{1}$ and $L_{y}=-r_{1}$, where $r_{1}$ denotes the constant radius of the central body.

\section{Wings}

The wings are modeled as thin, flat plates with a constant chord. The wing semispan ( $b$, the span of each wing) is set at $51.9 \mathrm{~mm}$. With an aspect ratio of 5.65 for both wings, the chord is set at $18.4 \mathrm{~mm}$. The wings are mounted at the wing joints at the midpoint of the wing, such that the center of mass of the wing is along the $y$ axis of the respective wing frame. The inertia tensors for the wings are calculated at the wing joint according to (using the right wing as an example)

$$
\mathbf{I}_{2, w}=\left[\begin{array}{ccc}
\frac{m_{2}}{3} b_{2}^{2} & 0 & 0 \\
0 & \frac{m_{2}}{12} c_{2}^{2} & 0 \\
0 & 0 & m_{2}\left(\frac{1}{3} b_{2}^{2}+\frac{1}{12} c_{2}^{2}\right)
\end{array}\right]
$$

The inertia tensor for the left wing, $\mathbf{I}_{3, w}$, is identical to the inertia tensor for the right wing in the wing frames. For aerodynamic force and moment calculations, the thickness of the wings is set at $0.076 \%$ of the wing semispan. The mass of each of the wings is set at $47 \mathrm{mg}$. The inertia tensor for the wing in Eq. (44) is expressed in the wing frame. To express the inertia tensor in the stroke plane frame, the inertia tensors in the wing frames are transformed according to

$$
\mathbf{I}_{2, \mathrm{sp}}=\mathbf{R}_{R}^{T} \mathbf{I}_{2, w} \mathbf{R}_{R} \quad \text { and } \quad \mathbf{I}_{3, \mathrm{sp}}=\mathbf{R}_{L}^{T} \mathbf{I}_{3, w} \mathbf{R}_{L}
$$

When the stroke plane is nonzero, the inertia tensors in the stroke plane frames are transformed to the $B$ frame according to

$$
\mathbf{I}_{2}=\mathbf{R}_{\beta_{R}}^{T} \mathbf{I}_{2, \mathrm{sp}} \mathbf{R}_{\beta_{R}} \quad \text { and } \quad \mathbf{I}_{3}=\mathbf{R}_{\beta_{L}}^{T} \mathbf{I}_{3, \mathrm{sp}} \mathbf{R}_{\beta_{L}}
$$

No further transformation of the inertia tensors is necessary, the derivation method only requires calculation of the inertia tensors with respect to the reference points for each rigid body.

\section{B. Aerodynamic Model}

The model used for the majority of the simulations is the quasisteady/blade-element model developed by Berman and Wang and presented in [12]. The model is slightly modified from that presented in $[12,26]$ in order to properly fit the presented dynamics. The reference directions are changed to fit our representation of the dynamics, e.g., from $r_{1}$ and $r_{3}$ in [26] to $r_{x}$ and $r_{z}$. Furthermore, notation is changed to fit with model development previously presented. The model includes linear and circulation terms, but does not include leading edge vortex or wake capture effects, which have been previously shown through CFD results to enhance lift. The morphological parameters for the FWMAV simulations to be presented are based off of a hawkmoth. In [12], Berman and Wang use the drag coefficients obtained by Usherwood and Ellington in [39]. Usherwood and Ellington used model hawkmoth wings, scaled to $0.5 \mathrm{~m}$, at a Reynolds number of 8071 , to obtain the drag coefficients used in the simulations.

The Berman and Wang model is chosen for the aerodynamic model because the drag coefficients are calculated for a Reynolds number equivalent to the Reynolds number of the wings in the simulation results. Although the model does not include wake capture effects or vortex sheddings effects, Sane and Dickinson determined in [16] that as high as $80 \%$ of the lift and drag generated in a hover condition are due to translation and rotational effects. Hedrick and Daniel state in [38] that a computationally efficient model for aerodynamics, including wake and vortex effects, is not presently available. The Ansari quasi-steady aerodynamic model, presented in $[40,41]$, includes wake and vortex effects, but high fidelity runs are not computationally efficient for control studies. Quasi-steady aerodynamic models have been used in multibody simulations presented in $[22,23,25,26]$. The intent of this study is to
Table 2 Wing parameter summary

\begin{tabular}{lccrrc}
\hline \hline Specimen & Semispan & Chord & $\begin{array}{c}x \text { position } \\
\text { of c.p. }\end{array}$ & $\begin{array}{r}y \text { position } \\
\text { of c.p. }\end{array}$ & $\begin{array}{c}\text { Reynolds } \\
\text { number }\end{array}$ \\
\hline Right wing & $51.9 \mathrm{~mm}$ & $18.4 \mathrm{~mm}$ & $4.6 \mathrm{~mm}$ & $29.6 \mathrm{~mm}$ & 8000 \\
Left wing & $51.9 \mathrm{~mm}$ & $18.4 \mathrm{~mm}$ & $4.6 \mathrm{~mm}$ & $-29.6 \mathrm{~mm}$ & 8000 \\
\hline
\end{tabular}

provide the basis for dynamics studies from a control standpoint. Therefore, a complicated, complex aerodynamic model with a large computation time will not result in pertinent calculations. A summary table of the pertinent wing parameters, for aerodynamic calculations is shown in Table 2 . The positions of the aerodynamic centers of pressure are shown for their fixed position in the respective wing frames.

A velocity of a point on the wing is required to calculate the aerodynamic forces and moments. The velocity on a point of the wing, relative to the body, is calculated in the following manner. The position of a point along the center of the wing, expressed in the $B$ frame, is given by

$$
\bar{r}_{\mathrm{RW}}=r \mathbf{R}_{\beta_{R}}^{T}\left[\begin{array}{c}
\cos \delta_{R} \sin \zeta_{R} \\
\cos \delta_{R} \cos \zeta_{R} \\
\sin \delta_{R}
\end{array}\right]
$$

for the right wing, where $r$ denotes the position along the wing in the stroke plane frame. The velocity of the wing is calculated according to the transport theorem such that

$$
\dot{\bar{r}}_{\mathrm{RW}}=\frac{\partial \bar{r}_{\mathrm{RW}}}{\partial t}+\bar{\omega}_{2} \times \bar{r}_{\mathrm{RW}}
$$

The acceleration of the wing is

$$
\ddot{\bar{r}}_{\mathrm{RW}}=\frac{\partial \dot{\bar{r}}_{\mathrm{RW}}}{\partial t}+\bar{\omega}_{2} \times \dot{\bar{r}}_{\mathrm{RW}}
$$

A similar procedure is used to develop the velocity and acceleration of the left wing. The angular velocities of the right and left wings, $\bar{\omega}_{2}$ and $\bar{\omega}_{3}$, are obtained from Eq. (19), which define the angular velocity of the wing with respect to the stroke plane frame. The force generation for a wing in the $x$ direction is

$$
d F_{x}=\left\{\left[\left(\frac{-c(r)}{\bar{c} R} m_{w}+m_{22}\right) \dot{\alpha}+\rho \Gamma\right] \dot{\bar{r}}_{z}-m_{11} \ddot{\bar{r}}_{x}\right\} d r
$$

and in the $z$ direction is

$$
d F_{z}=\left\{\left[\left(\frac{-c(r)}{\bar{c} R} m_{w}+m_{11}\right) \dot{\alpha}+\rho \Gamma\right] \dot{\bar{r}}_{x}-m_{11} \ddot{\bar{r}}_{z}\right\} d r
$$

where $c(r)$ denotes the chord as a function of the radial position of the wing, $m_{11}$ and $m_{22}$ are added mass terms, $m_{w}$ is the mass of the wing, and $\Gamma$ is the circulation term. The added mass terms are calculated according to

$$
m_{11}=\frac{\pi}{4} \rho \bar{t}^{2} \quad \text { and } \quad m_{22}=\frac{\pi}{4} \rho c(r)^{2}
$$

The circulation term is calculated according to the equation

$$
\Gamma=-\frac{1}{2} C_{T} c(r)\left\|\dot{\bar{r}}_{W}\right\|_{2} \sin (2 \alpha)+\frac{1}{2} C_{R} c(r)^{2} \dot{\alpha}
$$

The viscous effects on the wings are calculated by

$$
d F_{x}^{v}=\frac{1}{2} \rho c(r)\left(C_{D_{o}} \cos ^{2} \alpha+C_{D_{\pi / 2}} \sin ^{2} \alpha\right) \| \dot{\bar{r}}_{{ }_{2}} \dot{\bar{r}}_{x}
$$

and

$$
d F_{z}^{v}=\frac{1}{2} \rho c(r)\left(C_{D_{o}} \cos ^{2} \alpha+C_{D_{\pi / 2}} \sin ^{2} \alpha\right) \| \dot{\bar{r}}_{{ }_{2}} \dot{\bar{r}}_{z}
$$


$C_{T}, C_{D_{o}}$, and $C_{D_{\pi / 2}}$ are coefficients to fit the lift and drag of each wing. The constants used for simulation purposes are those for the hawkmoth presented in [12] and are obtained from experiments on model hawkmoth wings presented in [39]. The method does not calculate lift and drag directly, but the resultant forces in the $x$ and $z$ directions in the wing frames. The normal and tangential forces on the wings are calculated according to

$$
d F_{T}=d F_{x}-d F_{x}^{v} \quad \text { and } \quad d F_{N}=d F_{z}-d F_{z}^{v}
$$

For simulation purposes, five slices of the wing are used at each time step to integrate and calculate the resultant normal and tangential forces. Five slices are chosen based on the results presented in [38]. The lift, drag, and lateral forces generated by each wing in the $B$ frame are calculated according to Eq. (33). The resultant pitch, roll, and yaw moments are calculated using the results from Eqs. (33) and $(\underline{36})$.

\section{Results}

\section{A. Simulation Parameters}

The wing kinematics are based off of the biological flight mechanics of specimen F1 in [3]. The stroke plane is set at an angle of $16 \mathrm{deg}$ from the longitudinal axis of the central body. Because a positive rotation about the $\hat{b}_{y}$ being considered nose up, the angles of the stroke planes are $\beta_{R}=\beta_{L}=-16^{\circ}$. The deviation angle of the right and left wings is set at

$$
\delta(t)=\delta_{o}+\delta_{m} \sin \left(2 \pi N_{\delta} f t\right)
$$

The parameters $N_{\delta}$ is a shape parameter, which is set to two to ensure a proper figure-eight pattern for the wings. The specimen F1 has an average deviation angle of $0.9 \mathrm{deg}$. As a result, $\delta_{m}$ will be set to $1 \mathrm{deg}$ to approximate the kinematic pattern of hawkmoth. The sweep angle will be governed by the equation

$$
\zeta(t)=\zeta_{o}+\zeta_{m} \sin (2 \pi f t)
$$

Hawkmoths, like most insect species, generally exhibit a total flapping (stroke) amplitude of approximately $120 \mathrm{deg}$, therefore $\zeta_{m}$ will be set to $60 \mathrm{deg}$.

\section{B. Dynamic Model Comparison: Water-Treading Mode}

The water-treading mode is exhibited by insects when the angle of attack equals $0 \mathrm{deg}$ at the end of the upstroke and downstroke. A depiction of the angle of attack in the stroke plane is shown in Fig. 3 . The motion of the angle of attack is described by the motion:

$$
\alpha(t)=\alpha_{m} \sin \left(2 \pi f t+\Phi_{\alpha}\right)
$$

where $\Phi_{\alpha}$ is a phase shift to ensure the proper midstroke angle of attack. For the simulations presented, the amplitude is set at $45 \mathrm{deg}$ and the phase shift is set at $\pi / 2$. A phase shift of $\pi / 2$ ensures that the angle of attack is positive during the down stroke and that the midstroke angle of attack is $45 \mathrm{deg}$. Additionally, the angle of attack will be -45 deg during the upstroke, but since the wing is moving in
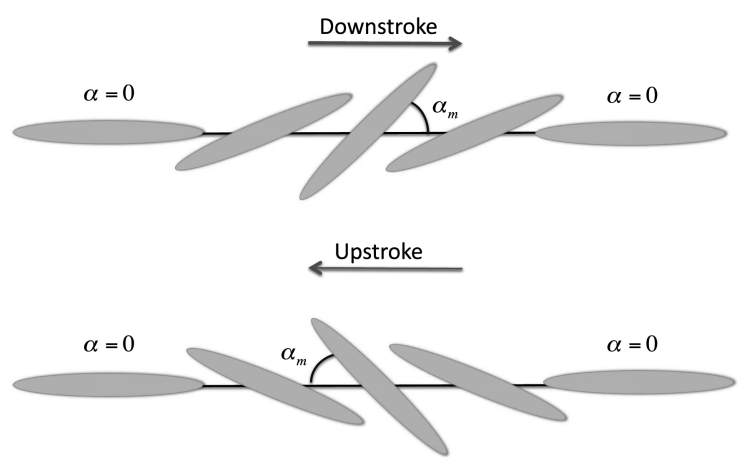

Fig. 3 Water-treading hovering mode.

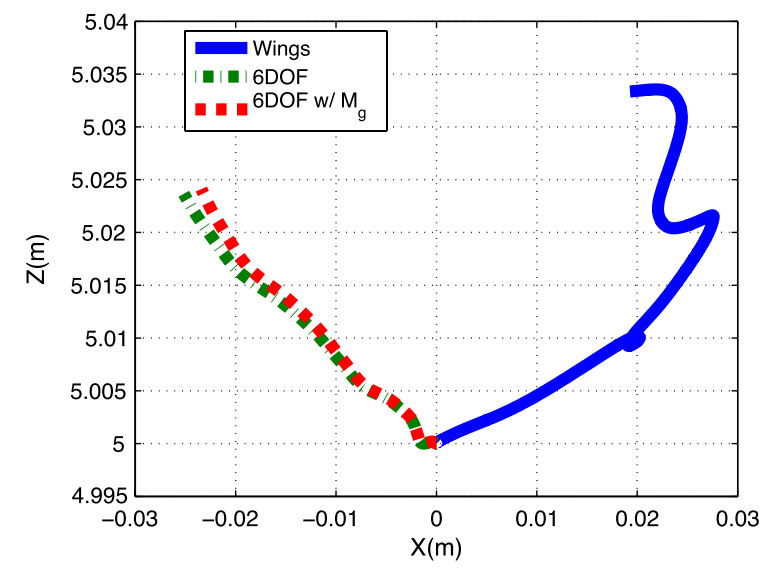

Fig. $4 \quad X-Z$ inertial position for water treading.

the opposite direction, the magnitude of the angle of attack is still correct. A phase of $3 \pi / 2$ will have the wing rotated in the wrong direction (negative on the downstroke, positive on the upstroke) and produce positive lift, quickly driving the vehicle into the ground. It is important to note that during the upstroke, the center of pressure of the wing is at a $x$ coordinate of $-(c / 4)$ in the wing frame since the wing is "flipped" over. The angle of attack presented in $[\underline{20}, \underline{21}]$ uses a water-treading mode. Simulations, for three flapping cycles, are presented in Figs. $\underline{4}$ and $\underline{5}$. For each of the plots, the solid line represents the full nonlinear model, the dotted line represents the standard aircraft model, and the dashed line is standard model with the addition of the gravity moments of the wings. The FWMAV starts at an initial height of $5 \mathrm{~m}$ with a pitch angle of $34.9 \mathrm{deg}$. The inertial plot, in Fig. 4, shows for a flapping frequency of $26 \mathrm{~Hz}$, the inertial effects of the wings push the vehicle away from the initial starting point and the vehicle gains altitude. The total lift, in the absence of inertial coupling effects, is enough for the standard aircraft models to slowly gain altitude. With no control, the resultant drag (thrust) forces pushed the vehicle models away from a hover condition. Figure 5 shows the pitch angle and velocity of the FWMAV simulations. There are great differences between the full nonlinear model and the standard aircraft models. The wings are started, for simulation purposes, at midstroke of the downstroke. The initial gravity moment is nose down, while the initial aerodynamic pitching moment is nose up. The gravity moment of the wings reduces some of the effects of the aerodynamic pitching moment on the central body, but the effect is small. In [28], the authors estimate that the peak-to-peak displacement of the center of mass of the hawkmoth in a hover condition is $4 \mathrm{~mm}$. Furthermore, Hedrick and Daniel in [38] found the maximum deviation of a hawkmoth center of mass in a true hover condition is $6.5 \mathrm{~mm}$. The simulations presented here are open loop, and not exhibiting a controlled, true hover condition. An average horizontal displacement of approximately $13 \mathrm{~mm}$ per flapping cycle is qualitatively consistent with the previous studies. The angular velocity of the three simulations is presented in Fig. 5 b. The simulations show a marked difference for the standard aircraft model with the absence of the gravity moments of the wings. The simulations show a steadily increasing magnitude in the pitch velocity, consistent with the results presented by Bolender in [26]. The standard aircraft model and the model with gravity moments included show a small difference in simulation results when compared with each other, but marked differences with the full nonlinear model.

\section{Dynamic Model Comparison: Aerodynamic Model Comparison}

The following simulations will show that the qualitative performance of the flight dynamics models, for both the model presented in this paper and the standard aircraft model, is similar for different aerodynamic models. The two flight dynamics models presented in the paper will be compared with different aerodynamic models: the Berman and Wang model previously discussed and the quasi-steady/blade-element model used in $[20,21]$. The model in 


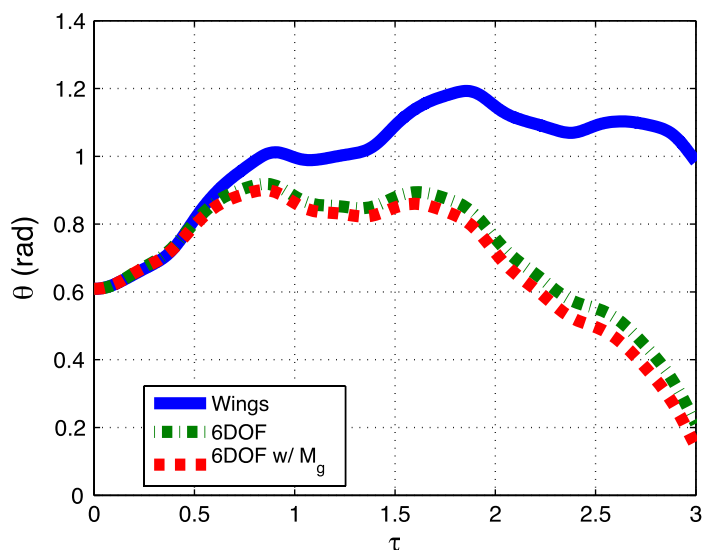

a) $\theta$ - pitch angle

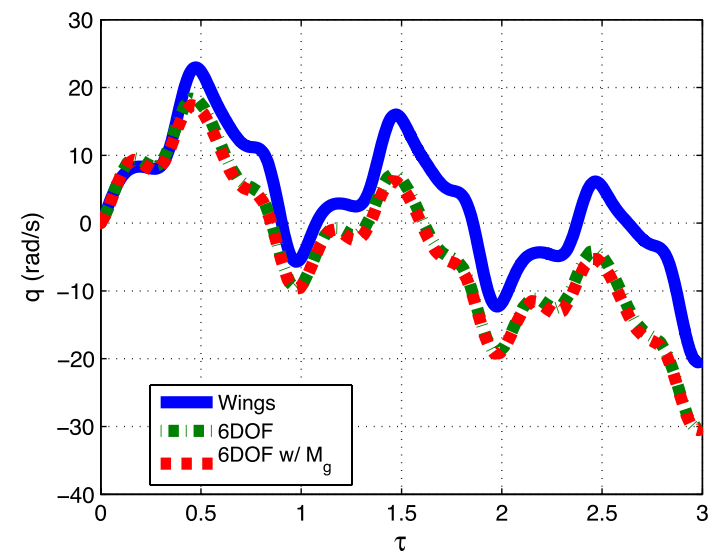

b) $q$ - pitch velocity

Fig. 5 Pitch motion for water treading.

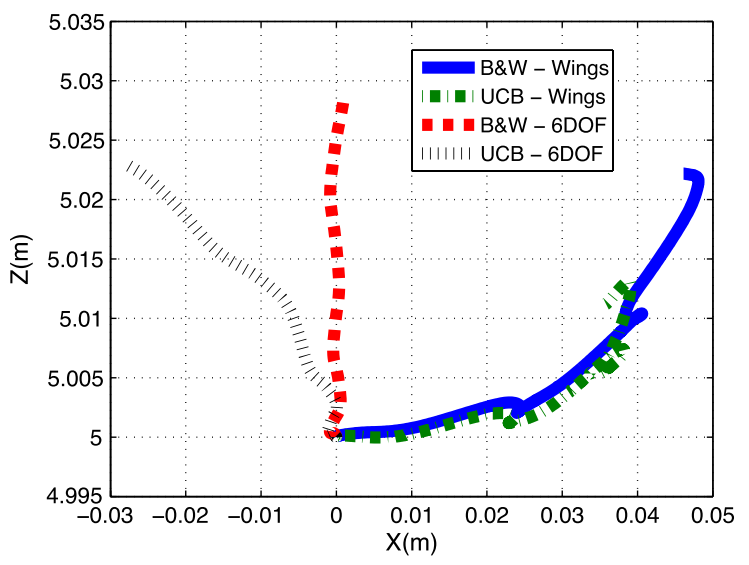

Fig. $6 X-Z$ inertial position for aerodynamic model comparison.

$[20,21]$ is a combination of a quasi-steady model from the Sane and Dickinson Robot Fly experiments and empirically matched data. The model includes delayed stall and rotational lift effects, but does not include wake capture or leading edge vortex effects. The model calculates normal and tangential forces on the wing. The forces due to the translation of the wing are

$$
\begin{gathered}
F_{\mathrm{tr}, N}(t)=\frac{1}{2} \rho A_{w} C_{N}(\alpha(t)) U_{\mathrm{cp}}^{2}(t) \quad \text { and } \\
F_{\mathrm{tr}, T}(t)=\frac{1}{2} \rho A_{w} C_{T}(\alpha(t)) U_{\mathrm{cp}}^{2}(t)
\end{gathered}
$$

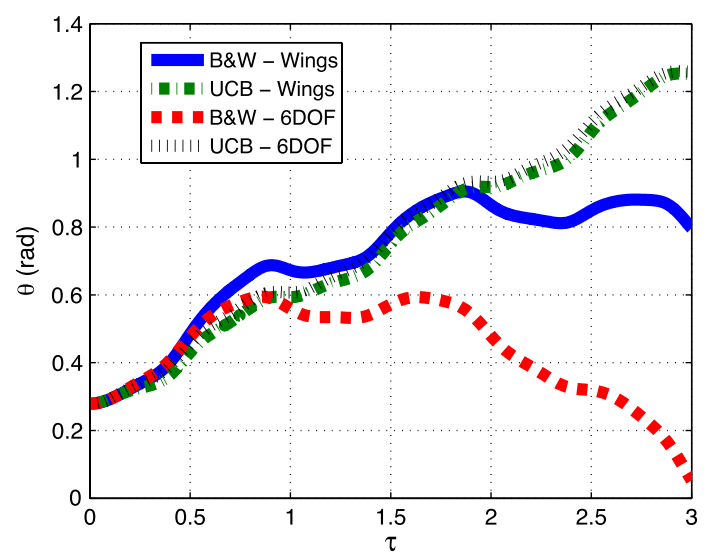

a) $\theta$ - pitch angle
The normal force on the wing, due to the rotation of the wing, is

$$
F_{\mathrm{rot}, N}(t)=\frac{1}{2} \rho A_{w} C_{\mathrm{rot}} c_{\mathrm{max}} \dot{\alpha}(t) U_{\mathrm{cp}}(t)
$$

In Eqs. (60) and (61), $A_{w}$ is the area of the wing, $C_{N}$ and $C_{T}$ are the normal and tangential force coefficients, $U_{\mathrm{cp}}(t)$ is the velocity of the center of pressure and $C_{\mathrm{rot}}$ is the rotational lift coefficient. The normal and tangential forces are transformed into the $B$ frame according to Eq. (33).

The comparison simulations are presented in Figs. 6 and 7. The simulation of the model with wing effects and the Berman and Wang model is represented by the solid line. The simulations of the full nonlinear model with the comparison aerodynamic model, denoted $\mathrm{UCB}$, is shown by the dash-dot line. The standard aircraft simulation results with the Berman and Wang model are represented by the dashed line and the results with the comparison model are represented by the dashed line. Simulations with the multibody model are denoted by Wings. Simulations with the standard aircraft model are denoted by $6 D O F$. The presented simulations are for a wing mass of $47 \mathrm{mg}$ per wing. The stroke plane angle is set at $-16^{\circ}$ and the initial pitch angle is $16^{\circ}$. The initial orientation of the wings is parallel to the ground in the inertial frame. The inertial position shows a qualitative similarity between the two models. The models with wing effects exhibit similar dynamic behavior, independent of the aerodynamic model. Likewise, the standard aircraft model exhibits similar behavior with different aerodynamic models. Both models produce a quantitative difference in position after three wing strokes. The aerodynamic models produce qualitatively similar behavior in the angular velocity of the central body. However, the quantitative difference is significant and produces different behavior.

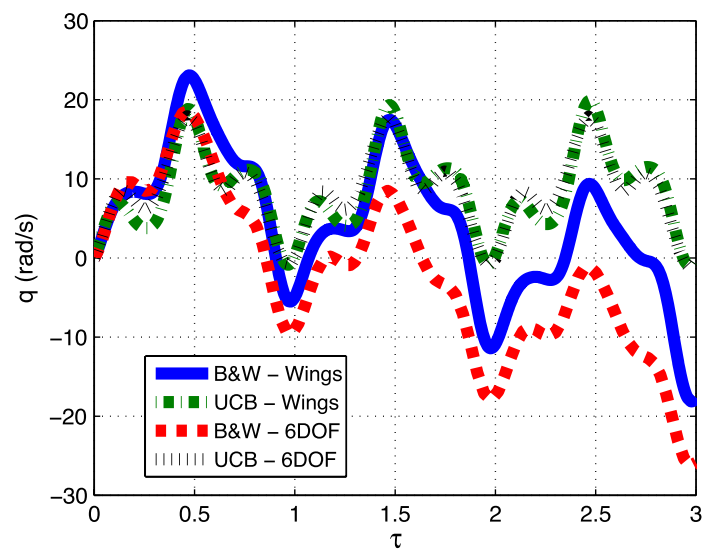

b) $q$ - pitch velocity

Fig. 7 Pitch motion for aerodynamic model comparison. 


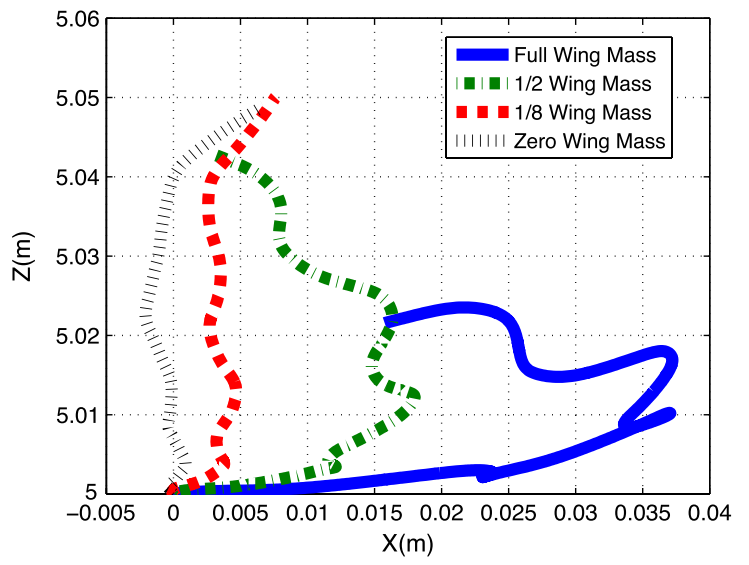

Fig. $8 \quad X$-Z inertial position for water treading with reduced wing mass.

The pitch angle of the model with wing effects increases at a faster rate with the UCB model than with the Berman and Wang model. A similar result is exhibited for the standard aircraft models. The UCB model does not predict a significant difference in the attitude of the FWMAV when the wing effects are included, by the position still exhibits a large difference, on the order of 1.5 body lengths in three flapping cycles. The Berman and Wang aerodynamic model predicts different behavior in both position and orientation, when the wing effects are included. The UCB model exhibits similar behavior in attitude, but different behavior in position, when the wing effects are included. In fact, the difference between the position of the two models for the UCB model is approximately $50 \%$ greater than that of the two dynamics models when the Berman and Wang model is used.

\section{Dynamic Model Comparison: Decreased Wing Mass}

The following simulations results show the comparison between the nonlinear model with wing effects and the standard aircraft model with the mass of the wings decreased from the previous watertreading simulations. The simulations use the same initial conditions and flapping frequency as the previously presented results. The aerodynamic model used is the model presented in [12]. Each set of simulations presented shows results for four flapping cycles. The initial pitch angle is $10^{\circ}$ and the stroke plane angle is $-10^{\circ}$. The first set of simulations show the inertial position, pitch angle and pitch velocity with the wing mass reduced by one half to $23.5 \mathrm{mg}$ per wing. The second set of comparison simulations presents results with the wing mass reduced to $1 / 8$ of the initial wing mass, or approximately $5.875 \mathrm{mg}$ for each wing. The following figures present a comparison between the simulations with the full wing mass, $1 / 2$ wing mass, $1 / 8$ wing mass, and zero wing mass. In the figures, the solid line represents the full wing mass. The $1 / 2$ wing mass is represented by the dash-dot line and the $1 / 8$ wing mass is represented by the dashed line. Finally, the simulation results for zero wing mass are represented by the dotted line.

The simulation results presented in Figs. $\underline{8}$ and 9 show that as the mass of the wings relative to the central body decreases, the simulation results from the full nonlinear model approach the results of the model with simple nonlinear dynamics. However, there are still differences in simulation results when the wings total only $0.71 \%$ of the total body mass, especially in the inertial position of the central body. The simulations presented with full wing mass showed that the wings may reduce the destabilizing effect of the aerodynamic pitching moment. As the mass of the wings is reduced relative to the central body, the aerodynamic pitching moment seems to have more of an effect on the attitude of the central body.

\section{Conclusions}

The paper presented the derivation and simulation of the nonlinear dynamics of a FWMAV with three degrees of freedom for each wing. The dynamic model includes the mass and inertia effects of the wings. The simulations show that the common practice of neglecting the mass of the wings produces a vastly different result from when some, or all, of the effects of the wings are included. Additionally, the choice of aerodynamic model, and the associated underlying assumptions, can make a substantial difference in the predicted behavior of the system. The total wing mass is $5.7 \%$ for the initial set of simulations, on the outside of the range of wing mass values that lead to neglecting the effects of the wings. However, the inertial position (and translational velocity) results are vastly different for the full nonlinear model and the standard aircraft models. For the same flapping frequency in a water-treading hovering mode, the full nonlinear model climbs and translates forward, while the standard aircraft models continue to ascend and translate backwards. Changing the aerodynamic model produces qualitatively similar results. A difference in behavior between the flight dynamics model with wing effects, and the model without, is present for a total wing mass of $0.7 \%$ of the body mass.

From a simulation standpoint, neglecting the mass effects of the wings on the central body of a FWMAV may be foolhardy for flight stability and controls studies of a flapping wing aircraft. Some, if not all, of the mass effects of the wings need to be included for a representative model of the aircraft dynamics and performance. The wing effects, as detailed in this study, are important in an open loop setting. When active controls are added to the system, the result may change. Furthermore, the relative importance of the effects of the wings on the motion of the central body needs to be examined. The inclusion of the wings as separate degrees of freedom and state variables may be important for future control studies. If the orientation of the wings is not part of the state, it may limit the availability of control options. The same analysis should be conducted with an aerodynamic model that includes vortex and wing effects. Future work will focus on simplification techniques for the dynamics, to

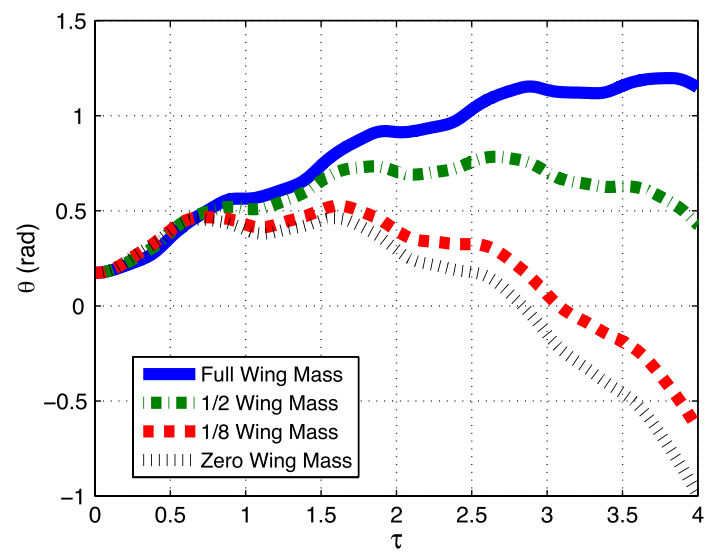

a) $\theta$ - pitch angle

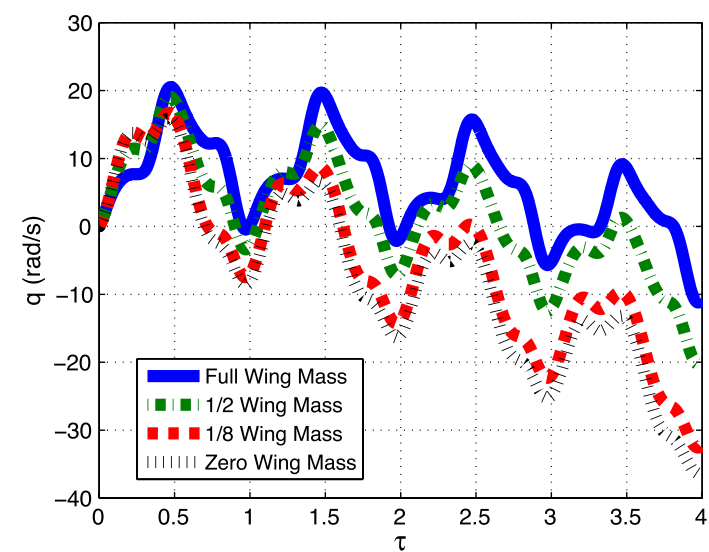

b) $q$ - pitch velocity

Fig. 9 Pitch motion for reduced wing mass. 
include the mass effects of the wings, that will allow for easier handling of the equations of motion, but still capture the important mass effects of the wings on the central body.

\section{References}

[1] Etkin, B., and Reid, L., Dynamics of Flight, Wiley, New York, 1996.

[2] Taylor, G., and Thomas, A., "Dynamic Flight Stability in the Desert Locust Schistocera Gregaria," Journal of Experimental Biology, Vol. 206, No. 16, 2003, pp. 2803-2829.

doi:10.1242/jeb.00501

[3] Willmott, A., and Ellington, C., "The Mechanics of Flight in the Hawkmoth Manduca Sexta," Journal of Experimental Biology, Vol. 200, No. 21, 1997, pp. 2705-2745

[4] Ellington, C., "The Aerodynamics of Hovering Insect Flight 1-4," Philosophical Transactions of the Royal Society of London, Vol. 305, No. 1122,1984 , pp. 1-113. doi:10.1098/rstb.1984.0049

[5] Wakeling, J., and Ellington, C., "Dragonfly Flight 1-3," Journal of Experimental Biology, Vol. 200, No. 3, 1997, pp. 543-600.

[6] Dudley, R., and Ellington, C., "Mechanics of Forward Flight in Bumblebees 1-2," Journal of Experimental Biology, Vol. 148, No. 1, 1990, pp. 19-88.

[7] Ellington, C., "The Novel Aerodynamics of Insect Flight: Applications to Micro Air Vehicles," Journal of Experimental Biology, Vol. 202, No. 23, 1999, pp. 3439-3448.

[8] Sun, M., Liu, P., and Wang, K., "Dynamic Flight Stability of a Hovering Hoverfly," New Trends in Fluid Mechanics: Proceedings of the Fifth International Conference on Fluid Mechanics, Aug. 15-19, 2007, Tsinghua Univ. Press, Shanghai, China, 2007, pp. 626-629.

[9] Taylor, G., Bomphrey, R., and Hoen, J., "Insect Flight Dynamics and Control," 44th AIAA Aerospace Sciences Meeting and Exhibit, AIAA Paper 2006-32, Reno, NV, 2006.

[10] Sun, M., and Xiong, Y., "Dynamic Flight Stability of a Hovering Bumblebee," Journal of Experimental Biology, Vol. 208, No. 3, 2005, pp. 447-549. doi: $10.1242 / \mathrm{jeb} .01407$

[11] Dietl, J., and Garcia, E., "Stability in Ornithopter Longitudinal Flight Dynamics," Journal of Guidance, Control, and Dynamics, Vol. 31, No. 4, July-Aug. 2008, pp. 1157-1162. doi: $10.2514 / 1.33561$

[12] Berman, G., and Wang, Z., "Energy-Minimizing Kinematics in Hovering Insect Flight," Journal of Fluid Mechanics, Vol. 582, July 2007, pp. 153-168. doi:10.1017/S0022112007006209

[13] Doman, D., Oppenheimer, M., and Sigthorrson, D., "Dynamics and Control of a Minimally Actuated Biomimetic Vehicle: Part 1: Aerodynamic Model," AIAA Guidance, Navigation, and Control Conference, AIAA Paper 2009-6160, Chicago, IL, 2009.

[14] Doman, D., Oppenheimer, M., and Sigthorrson, D., "Dynamics and Control of a Minimally Actuated Biomimetic Vehicle: Part 2: Control," AIAA Guidance, Navigation, and Control Conference, AIAA Paper 2009-6161, Chicago, IL, 2009.

[15] Wood, R., "The First Takeoff of a Biologically Inspired at-Scale Robotic Insect," IEEE Transactions on Robotics and Automation, Vol. 24, No. 2, 2007, pp. 341-347. doi:10.1109/TRO.2008.916997

[16] Sane, S., and Dickinson, M., "The Control of Flight Force by a Flapping Wing: Lift and Drag Production," Journal of Experimental Biology, Vol. 204, No. 15, 2001, pp. 2607-2626.

[17] Khan, Z., and Agrawal, S., "Modeling and Simulation of Flapping Wing Micro Air Vehicles," ASME International Design Engineering Technical Conference, American Society of Mechanical Engineers, Long Beach, CA, pp. 871-879.

[18] Deng, X., Schenato, L., and Sastry, S., "Hovering Flight Control of a Micromechanical Flying Insect," 40th IEEE Conference on Decision and Control, Institute of Electrical and Electronics Engineers Paper TuA08-5, Orlando, FL, 2001.

[19] Schenato, L., Campolo, D., and Sastry, S., "Controllability Issues in Flapping Flight for Biomimetic Micro Air Vehicles (MAVS)," 42nd IEEE Conference on Decision and Control, Institute of Electrical and Electronics Engineers Paper FrE10-5, Maui, HI, 2003.

[20] Deng, X., Schenato, L., Wu, W., and Sastry, S., "Flapping Flight for Biomimetic Robot Insects: Part 1: System Modeling," IEEE Transactions on Robotics and Automation, Vol. 22, No. 4, Aug. 2006, pp. 776-788.

doi: $10.1109 /$ TRO. 2006.875480
[21] Deng, X., Schenato, L., and Sastry, S., "Flapping Flight for Biomimetic Robot Insects: Part 2: Flight Control Design," IEEE Transactions on Robotics and Automation, Vol. 22, No. 4, Aug. 2006, pp. 789-803. doi:10.1109/TRO.2006.875483

[22] Lasek, M., and Sibilski, K., "Modeling and Simulation of Flapping Wing Control for a Micromechanical Flying Insect (Entomopter)," AIAA Modeling and Simulation Technologies Conference and Exhibit, AIAA Paper 2002-4973, Monterrey, CA, 2002.

[23] Buler, W., Loroch, L., Sibilski, K., and Zyluk, A., "Modeling and Simulation of the Nonlinear Dynamic Behavior of a Flapping Wings Micro-Aerial-vehicle," 42nd AIAA Aerospace Sciences Meeting and Exhibit, AIAA Paper 2004-541, Reno, NV, 2004.

[24] Jackson, J., Bhattacharya, R., and Strganac, T., "Modeling and Suboptimal Trajectory Generation for a Symmetric Flapping Wing Vehicle," AIAA Guidance, Navigation, and Control Conference and Exhibit, AIAA Paper 2008-6465, Honolulu, HI, 2008.

[25] Grauer, J., and Hubbard, J., "Multibody Model of an Ornithopter," AIAA Journal of Guidance, Control, and Dynamics, Vol. 32, No. 5, 2009, pp. $1675-1679$. doi:10.2514/1.43177

[26] Bolender, M. A., "Rigid Multi-Body Equations-of-Motion for Flapping Wing MAVs Using Kane's Equations," AIAA Guidance, Navigation, and Control Conference, AIAA Paper 2009-6158, Chicago, IL, 2009.

[27] Sun, M., Wang, J., and Xiong, Y., "Dynamic Flight Stability of Hovering Insects," Acta Mechanica Sinica, Vol. 23, No. 3, 2007, pp. 231-246. doi:10.1007/s10409-007-0068-3

[28] Wu, J., Zhang, Y. L., and Sun, M., "Hovering of Model Insects: Simulation by Coupling Equations of Motion with Navier-Stokes Equations," Journal of Experimental Biology, Vol. 212, No. 20, 2009, pp. 3313-3329. doi:10.1242/jeb.030494

[29] Gebert, G., Gallmeier, P., and Evers, J., "Equations of Motion for Flapping Flight," AIAA Atmospheric Flight Mechanics Conference, AIAA Paper 2002-4872, Monterrey, CA, 2002.

[30] Dickson, W., Straw, A., Poelma, C., and Dickinson, M., "An Integrative Model of Insect Flight Control," 44th AIAA Aerospace Sciences Meeting and Exhibit, AIAA Paper 2006-34, Reno, NV, 2006.

[31] Shyy, W., Lian, Y., Tang, J., Viieru, D., and Liu, H., Aerodynamics of Low Reynolds Number Flyers, Cambridge Univ. Press, New York, 2008.

[32] Sun, M., and Du, G., "Lift and Power Requirements of Hovering Insect Flight," Acta Mechanica Sinica, Vol. 19, No. 5, 2003, pp. 458-469. doi:10.1007/BF02484580

[33] Greenwood, D. T., Advanced Dynamics, Cambridge Univ. Press, New York, 2006.

[34] Greenwood, D. T., Principles of Dynamics, Prentice-Hall, Upper Saddle River, NJ, 1988.

[35] Baruh, H., Analytical Dynamics, McGraw-Hill, Boston, MA, 2006.

[36] Orlowski, C., Girard, A., and Shyy, W., "Derivation and Simulation of the Nonlinear Dynamics of a Flapping Wing Micro-Air Vehicle," 2009 European Micro-Air Vehicle Conference and Competition, Delft, The Netherlands, 2009.

[37] Orlowski, C., Girard, A., and Shyy, W., "Open Loop Pitch Control of a Flapping Wing Micro Air Vehicle Using a Tail and Control Mass," 2010 American Control Conference, Institute of Electrical and Electronics Engineers, Baltimore, MD, 2010, pp. 536-541.

[38] Hedrick, T., and Daniel, T., "Flight Control in the Hawkmoth Manduca Sexta: The Inverse Problem of Hovering," Journal of Experimental Biology, Vol. 209, No. 16, 2006, pp. 3114-3130. doi:10.1242/jeb.02363

[39] Usherwood, J., and Ellington, C., "The Aerodynamics of Revolving Wings 1: Model Hawkmoth Wings," Journal of Experimental Biology, Vol. 205, No. 11, 2002, pp. 1547-1564.

[40] Ansari, S., Zbikowski, R., and Knowles, K., "Non-Linear Unsteady Aerodynamic for Insectlike Flapping Wings in the Hover: Part 1: Methodology and Analysis," Journal of Aerospace Engineering, Vol. 220, No. 2, 2006, pp. 61-83. doi:10.1243/09544100JAERO049

[41] Ansari, S., Zbikowski, R., and Knowles, K., "Non-Linear Unsteady Aerodynamic for Insectlike Flapping Wings in the Hover: Part 2: Implementation and Validation," Journal of Aerospace Engineering, Vol. 220, No. 2, 2006, pp. 169-186. doi:10.1243/09544100JAERO050 